\title{
Tuning of Quantum Entanglement of a Superconductor by Transition-Metal and Rare-Earth Impurity Effect and the Role of Potential Scattering on Quantum Phase Transition
}

Neda Ebrahimian

Shahed University

Mehran Khosrojerdi

K.N.Toosi University of Technology

Reza Afzali ( $\square$ afzali@kntu.ac.ir)

K.N.Toosi University of Technology

\section{Research Article}

Keywords: Shiba-Rusinov model, electron spins, quantum phase transitions (QPTs), quantum correlation

Posted Date: June 3rd, 2021

DOI: https://doi.org/10.21203/rs.3.rs-564111/v1

License: (c) (i) This work is licensed under a Creative Commons Attribution 4.0 International License.

Read Full License 


\title{
Tuning of quantum entanglement of a superconductor by Transition-metal and Rare-earth impurity effect and the role of potential scattering on quantum phase transition
}

\author{
N. Ebrahimian', M. Khosrojerdi ${ }^{2}$, R. Afzali ${ }^{2 *}$ \\ ${ }^{1}$ Department of Physics, Faculty of Basic Sciences, Shahed University, Tehran, 3319118651, Iran \\ ${ }^{2}$ Department of Physics, K. N. Toosi University of Technology, Tehran, 15875-4416, Iran \\ Emails: n.ebrahimian@shahed.ac.ir, \\ khosrojerdi@alumni.kntu.ac.ir, \\ *Corresponding author: afzali@,kntu.ac.ir
}

\begin{abstract}
By considering transition-metal (Shiba-Rusinov model) and rare-earth metal impurities (Abrikosov-Gorkov theory) effect on a many-body system, i.e., a BCS s-wave superconductor, quantum bipartite entanglement of two electrons of the Cooper pairs in terms of the exchange interaction, $J$, the potential scattering, $V$ (playing an important role, unexpectedly), and the distance of two electron spins of the Cooper pair is calculated at zero temperature by using two-electron spin-space density matrix (Werner state). In transition-metal case, we find new quantum phase transitions (QPTs). The changes of $J$, which causes to have localized excited state, $V$ and the pairing interaction (via energy gap) lead to the displacement of the QPTs (interactions act in the same direction, however sometimes the pairing interaction causes the competition with other interactions), regardless of their effects on the value of concurrence. Determining the allowable values of all interactions by itself is not possible, due to the smallness of the perturbed Green's functions (appearing in the density matrix). For non-magnetic and magnetic (rare-earth) impurity cases, concurrence versus the distance and collision times is discussed for all finite and infinite Debye frequency. The quantum correlation, instability of the system and
\end{abstract}


what's more important QPT can be tuned by the impurity.

\section{Introduction}

During the last years, it became evident that quantum entanglement $(\mathrm{QE})$ is one of the most important resource in quantum information (QI) and quantum computation, especially, if QE emerges on a many-body system. The qualitative and quantitative aspects of QI revealed in the extensive research efforts by $\mathrm{QE}^{1-6}$. The method of measuring the entanglement is a broad field of research on its own. The consideration of the QI and QE in the many-body systems may lead to the revealing of the new entanglement properties ${ }^{7}$. It should be noted that the entanglement of many-body systems is extraordinarily complicated in comparison with other systems, especially, in the ground state permeate multipartite entanglement at zero temperature. The generation and manipulation of the bipartite and multipartite entangled states were investigated by many-body Hamiltonians ${ }^{7-}$

${ }^{12}$. Other investigations on many-body systems such as the entanglement of electron spins are as follows. The entanglement of two-electron spins of a non-interacting electron gas based on the Green's function approach has been discussed ${ }^{13-14}$; also, multipartite entanglement in a non-interacting Fermi gas was studied ${ }^{15}$. Furthermore, a few pieces of research have been done on superconductors using the space-spin density matrix approach. One of them is about bipartite entanglement of two-electron spins forming Cooper pairs in a BCS s-wave superconductor ${ }^{8}$; another one is about bipartite and tripartite entanglement and quantum correlation of s-wave and d-wave bulk and nano grain superconductors, which was given by some of our authors ${ }^{9,10,49}$.

In numerous works have been found that the entanglement, i.e., quantum correlations between subsystems, can be a good indicator of QPTs, which can be found in the many-body systems ${ }^{4,16}$. A lot of works have revealed that the bipartite (pairwise entanglement) either itself or its derivatives displays local extremum close to quantum critical points (QCPs). It merits mentioning that basically, the investigation of the entanglement on its own isn't a necessary and sufficient condition for the investigation of the emerge of QPT; one-to-one correspondence between QPT and the appearance of the critical point on concurrence doesn't exist necessarily, unless under some conditions, which was usually used in some spin models such as the Ising model ${ }^{16-19}$. However, one-to-one correspondence between QPT (accompanied by discontinuities of the ground state) and the 
behavior of the matrix elements of the density matrix always exist. Nevertheless, in our present work, there is a one-to-one correspondence between QPT and entanglement.

Here, we bring about some theories related to the impurity effect on superconductors. Over the past decades, researchers and scientists have investigated the impurities effect on the properties of the superconductors ${ }^{20-22}$. Shiba, Rusinov, Abrikosov, and Gorkov have presented theories about the Green's functions and some physical properties of the superconductors in the presence of two kinds of the impurities ${ }^{23-27}$. Shiba and Rusinov independently, have given the theory about the presence of the low concentration of the uncorrelated transition-metal impurities into a superconductor. In Shiba-Rusinov (SR) model, the scattering is calculated exactly for a single impurity problem by treating the impurity spin classically and it has been concluded exist a localized excited state in the energy gap ${ }^{25-27}$. The use of the SR model on the isotropic s-wave superconductors doped with the magnetic atoms specifies that the information on the potential scattering and the exchange interaction cannot be separately obtained; also, the energy of the bound states is a function of them ${ }^{28}$. The effect of the impurities on the anisotropic superconductors has been investigated by SR model; furthermore, Josephson current and several other properties of the superconducting alloy have been studied by SR approach $^{29-33}$. Several valuable theoretical and experimental researches on the QPT in the superconductors in the presence of the magnetic impurity have done by using the lattice model, Bogoliubov-de-Gennes procedure and Majorana fermion approach ${ }^{34-41}$. The robustness of the isotropic s-wave superconductors in the presence of non-magnetic impurities, based on the symmetry of the system, was studied by Anderson ${ }^{23}$. The role of the rare-earth metal impurities case in the conventional superconductors was studied by Abrikosov and Gorkov $^{24}$.

The purpose of this paper is to investigate of QPTs (based on our knowledge, these are new QPTs) and the bipartite entanglement properties of the BCS s-wave superconductor, in the presence of the non-magnetic impurity and magnetic impurities (transition-metal accompanied with SR model and the rare-earth metal accompanied with Abrikosov-Gorkov theory) at zero temperature by using the space-spin density matrix, up to the first-order approximation. Meanwhile, the potential scattering, which plays an important role as similar as the role of the exchange interaction, is investigated on QE. It merits mentioning that the increase of the absolute value of the exchange interaction in the 
appearance of QPTs always causes the increase of the potential scattering; and basically the effects of $|J|$ and $V$ in the concurrence are always coupled. The values of all available parameters such as the coefficients of the interactions depend on the assumptions drastically, which are tuned by the numerical calculations. Chiefly, we consider two basic assumptions; first, the smallness of the perturbed Green's functions (in the presence of the impurity) in comparison with the unperturbed Green's functions (at the whole range of allowed distances between two electrons of the Cooper pair) and second, the smallness of the appeared parts caused by the impurities in the renormalized order parameter (or energy gap), and the single-particle energy and frequency.

In this work, first, we address the investigation of the role of the exchange interaction, potential scattering, and normalized transition-metal impurity concentration in the SR model by considering the entanglement approach. Furthermore, the appearance of the QPTs and their displacement are investigated based on the parameters, especially, potential scattering (regardless of the influence of the parameters on the value of concurrence). Contrary to expectations, we show that the potential scattering plays an important role in the SR model. Second, we investigate the effect of the rare-earth metal (except cerium) and non-magnetic impurities in the conventional superconductors up to the first-order approximation by considering the Abrikosov-Gorkov (AG) theory. One of the most important assumptions in the AG theory is that the exchange interaction between a conduction electron and a magnetic impurity spin is weak and the lowest-order Born approximation is used to treat the scattering. For both rare-earth metal and non-magnetic impurities, we address to the investigation of both infinite and finite Debye frequencies. We find that the comparison among the finite Debye frequency, $\omega_{D}$, the order parameter (energy gap), $\Delta$, and the frequency, $\omega$, makes different conditions on the analytical and numerical calculations for Green's functions and subsequently, their appropriate values for the concurrence. Also, in the presence of the rare-earth impurity, for the infinite Debye frequency case, we have worked on the perturbed Green's functions up to the first approximation (it should be mentioned that previously, just Green's functions have been considered with infinite Debye frequency ${ }^{21}$ ). 


\section{Results and discussion}

\section{A. The transition-metal impurity case}

First, we consider the effect of the transition-metal impurity on the s-wave superconductor, which is satisfied by the SR model. This effect is investigated with respect to the critical impurity concentration, exchange interaction and the potential scattering, which has an important role in quantum correlation such as QE. It merits mentioning that unexpectedly, not only the potential scattering as well as the exchange interaction plays the important role in concurrence, but also, the existence of the QPTs depends on the presence of the potential scattering. Furthermore, of course, if exchange interaction is non-zero, then, the potential scattering becomes important term to appear the QPTs; otherwise, if the exchange interaction is zero, the potential scattering acts like non-magnetic impurity in the AG theory. Concurrence $\left(c \equiv c\left(p, j, v, k_{F} r\right)\right)$ in the presence of the impurity is calculated as a function of the internal and external parameters, i.e., $v$, which is related to the potential scattering, $V$, via $V \equiv v / \pi N(0), j$, whichis defined by the exchange interaction, $J$, via $J \equiv 2 j / S \pi N(0), p$, which is defined as the normalized impurity concentration, and $k_{F} r$. Meanwhile, $N(0), S, r$ and $k_{F}$ are the density of single-particle states at the Fermi surface, the spin of the impurities, the distance of two electrons of the Cooper pairs and Fermi wave number, respectively. We study the effect of the variations of these parameters on concurrence and QPTs for the transition-metal impurity case (throughout the paper, we use $\hbar=1$ ).

Concurrence as a measure of bipartite entanglement in the presence of the transition-metal impurities versus $v$ at different fixed values of $p, k_{F} r$, and $j$ are depicted in Fig.1. We would like to consider Green's functions up to the first-order approximation for calculating concurrence, so the ratio of the perturbed Green's functions to the primary Green's functions must be always controlled to have a small value. Therefore, by decreasing the $|j|$, the appropriate range of the values of $v$ becomes more limited to satisfy our approximation, so that for any step of our calculation, the situation must be checked. Almost, by considering the high values of $|j|$ and the lower value of $p$ we have a full range of $v$. It should be mentioned that different fixed values of $k_{F} r$ will not affect the restrictions over the values of $v$ to meet our approximation. The appearance of the critical 
points (such as the turning point and the local maximum point) depends on the $j$ values. Of course, some special values of $j$ have been chosen so that the turning point and the local maximum point appear. Also, it should be mentioned that the only effect of different fixed values of $p$ is the change of the value of concurrence. As it can be seen from Fig.1, by increasing the normalized impurity concentration at the fixed value of $v$, concurrence increases. In the inset of Fig.1, concurrence versus $v$ has been plotted in the presence of transition-metal impurities for different fixed values of $k_{F} r$ at a fixed $p$ and a fixed $j$. As expected, by increasing the relative distance between two electrons of a Cooper pair of a conventional superconductor (or an s-wave superconductor), concurrence reduces. The behavior of the curves of the inset is similar to each other and all critical points occur at the same value of $v$. The curves in Fig. 1 has a turning point that appears in the curve route and due to the changing the concavity from down to the up. For each $v$ that located in the almost interval $(0.70,0.95)$ with $|j|=0.8$, curves are completely ascending.

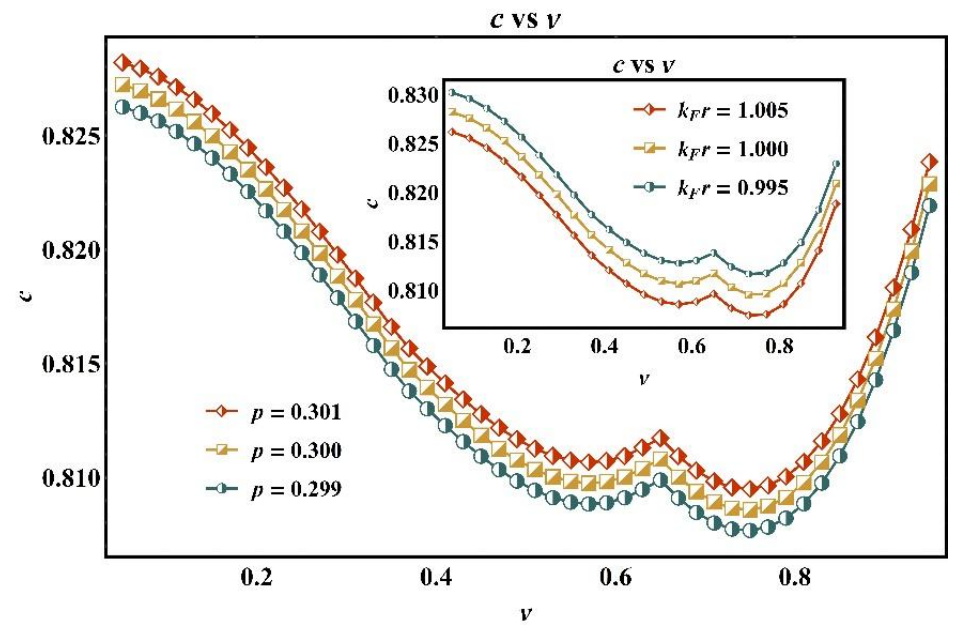

Figure 1. For transition-metal case; concurrence versus $v$ for various values of the $p$ at $j=-0.8$ and $k_{F} r=1$. Inset: concurrence versus $v$ for various values of the $k_{F} r$ at $j=-0.8$ and $p=0.3$.

In Fig.2. concurrence versus $v$ is depictedat different fixed values of $j$, at a fixed value of the relative distance and a fixed value of the normalized impurity concentration. Intentionally, the curves have been brought in Fig.2, because of showing how to disappear one of the local maximum points (QCP) with reducing the absolute value of the exchange interaction. By using the derivatives of concurrence, we find that the annihilation of the critical point occurs about $j \approx-0.60$ (we have considered the typical value of the energy 
gap and Debye frequency). It is worth mentioning that the high value of $p$, with respect to our approximation, leads to the restriction on the appropriate range of the $v$ (the values of $p$ and $k_{F} r$ have been chosen 0.2 and 1, respectively). From Fig.2, the change of the value of $j$ leads to the change of concurrence and bipartite entanglement; the QCPs have been formed when the values of $j$ have been located almost between -0.60 and -0.99 . It seems that by reducing the value of $|j|$, these points are displaced into the left-hand side of the curves. For each $j$ located in the almost interval $(-0.05,-0.60)$, such as the curve identified with $j \approx-0.60$ in Fig.2, the curve does not show any critical point. For $j=$ -0.68 , in the interval $v \approx(0.05,0.4)$, the variation of concurrence wasn't noticeable, but, after passing the local maximum (minimum) point, concurrence decreases (increases) up to $v \approx 0.6(v \approx 0.95)$. By increasing the absolute value of $j$, both the turning point and the local maximum point move to the high value of the $v$ (move to the right-hand side on the $v$ axis).The turning point (the local maximum point) is disappeared at $j<|-0.64|(j<\mid-$ $0.60 \mid$ ). Of course, again it should be mentioned that this disappearance is influenced by the approximation used in all calculations. In addition, it can be seen that by increasing the absolute value of $j$, the concurrence decreases.

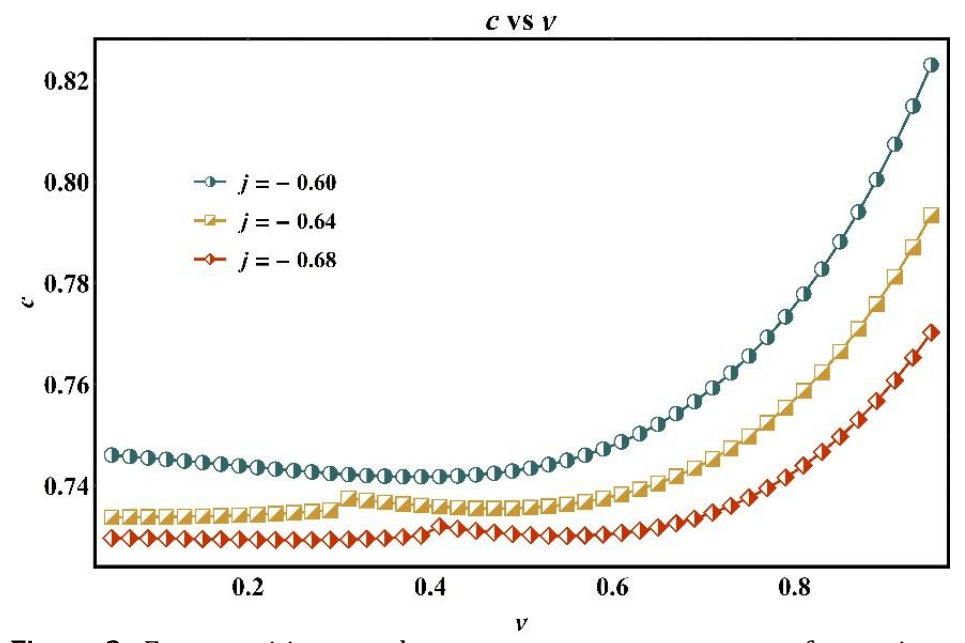

Figure 2. For transition-metal case; concurrence versus $v$ for various values of the $j$ at $p=0.2$ and $k_{F} r=1$.

It should be noted that in the presence of the magnetic impurity, the energy gap is reduced $^{25}$; this reduction depends on the normalized impurity concentration, the exchange interaction, the potential scattering and the density of states. Our calculations show that the variation of the superconducting energy gap (order parameter) due to the magnetic 
impurity is very small, so we can neglect the effect of this reduction on the energy gap and thereby, the maximum error occurred in the calculation is only a small percentage of it. Therefore, we used a typical value of the energy gap such as that given in Ref. [8].

However, for s-wave superconductors with all these descriptions, we address the investigation of the different values of the energy gap (and of the Debye frequency). We show that how the different values of the energy gap can affect not only the value of concurrence but also the occurrence of the critical points at different values of the exchange interaction and potential scattering. Concurrence versus vat different fixed values of the $\Delta$ is depicted in Fig.3. At different values of $\Delta$ or pairing interaction, the turning point and the local maximum point can be displaced and these points can be disappeared up to the first-order approximation; for example, by considering the specified values of $\Delta$ in Fig.3, the lowest curve doesn't show any the maximum local point. By increasing the value of $\Delta$, the turning point (the local maximum point) moves toward the lower (higher) value of the $v$. And eventually, it can be seen from Fig.3, by enhancing the value of $\Delta$, concurrence increases.

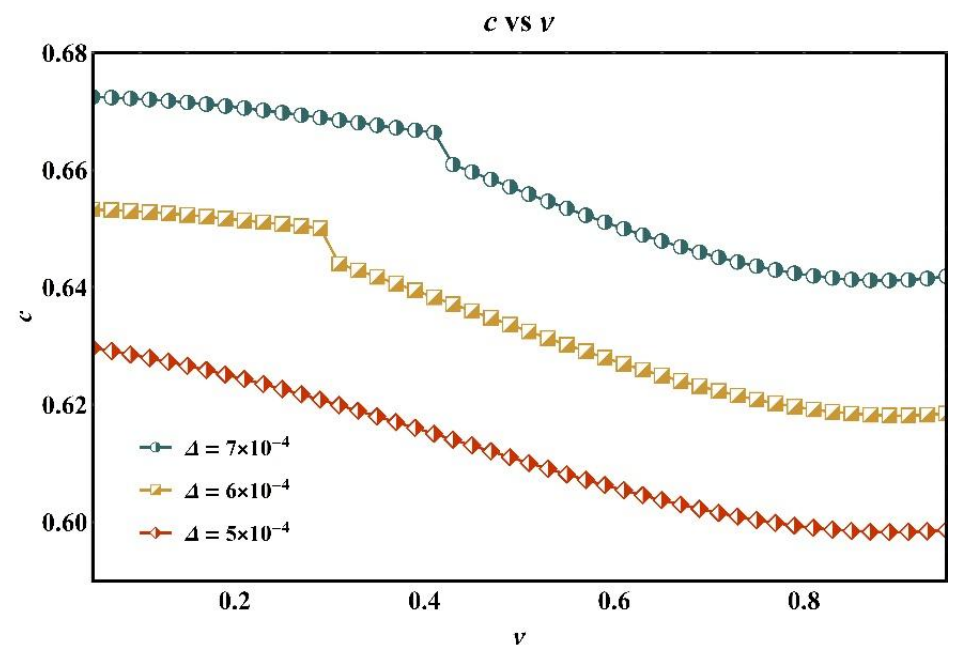

Figure 3. For transition metal case; concurrence versus $v$ for various values of the $\Delta$ at $j=-0.8, p=0.2$ and $k_{F} r=1$.

The study of the partial derivative of concurrence versus $v$ gives us the valuable information about the critical points such as the turning point and the local maximum point (we consider the typical value of $j=-0.8, k_{F} r=1$, and $p=0.3$ ). For addressing to this 
certain issue first we start to explain Fig.4(a). For each $v$ that located in the almost interval $(0.1,0.5)$, we find that the first partial derivative of $c$ versus $v$ has a minimum at $v \approx 0.29$, the value of $\partial_{v}^{2} c$ is equal zero and the value of the third partial derivative wasn't zero. Concurrence is continuous at $v \approx 0.29$ and also at this point, which is the turning point, the concavity of the curves is changed from down to up. Second, from Fig.4 (b), for each $v$ that located in the almost interval $(0.635,0.665), \partial_{v} c$ is equal zero at $v \approx$ 0.650 and the second partial derivative has a minimum; it can be seen that at this value of the $v$, the local maximum point appears and this point behaves as a critical point. We investigate the curves of $c$ vs $v$ in three regimes, at a fixed exchange interaction, from lower to the higher value of the potential scattering. The first regime contains the turning point (first concurrence reduces with the sharp slope, then the slope of the curve becomes reverse), the second regime is started from this point and is contained the local maximum point, finally, the third regime includes the curves that are quite ascending with respect to the potential scattering. We have concluded the emergence of the QPT points, by our analytical and numerical studies on concurrence (and also, via the energy of the system), its first and second derivatives. By changing the values of $j$ and $\Delta$, these critical points (turning point and local maximum point) emerge at different the $v$; also, the normalized impurity concentration and the relative distance do not affect on the displacement of QPT points, which will be discussed in the following.
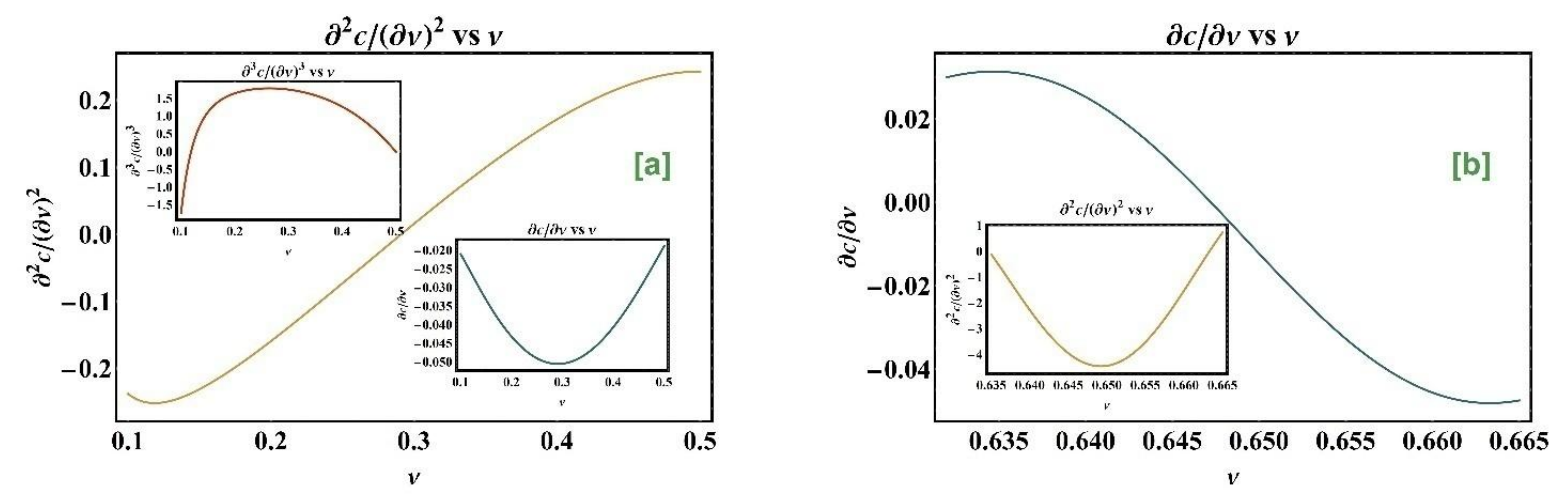

Figure 4. partial derivatives of $c$ vs vat $j=-0.8, k_{F} r=1$ and $p=0.3(\mathrm{a})$ : for each $v$ that located in the interval(0.1,0.5)(b): for each $v$ that located in the interval $(0.635,0.665)$.

Now, we proceed to interpret the change of the bipartite QE of two electron spins forming Cooper pairs. It should be mentioned that concurrence (and also, via the energy of 
the system) shows the existence of QPTs. There are the necessary and sufficient conditions for QCPs existing in Figs. 1 and 2 to describe as QPT points; we investigate the behavior of these points not only by the change of concurrence but also by the change of energy (it should be mentioned that because concurrence is our favorite subject in the exposition of the QPTs so we continue the interpretation of the changing of concurrence, of course, to ensure the accuracy of the QPTs point, we have investigated the condition of the changing of the energy at these points), and their first and second derivatives, exactly. In fact, in our work, there is a one-to-one correspondence between QPT and entanglement. The turning point and the local maximum point due to the existence of the potential scattering are new QPT points. It worth mentioning that when the cooper pair exists and bipartite entanglement is non-zero, the relative distance doesn't affect on the appearance or disappearance of the QPT points. Previously, the system has been shown the QPT, when the bound state is induced ${ }^{25-27}$; however, our investigation about QPTs (via QE) is associated with the values of the exchange interaction and the potential scattering that deal with other cases accompanied by new QPTs. It is worth mentioning that we know exactly what happens in the system and how we can see the interaction influences on the correlation of the electron spins of the Cooper pair. In the other words, it is very interesting that to follow the effect of the all real interactions (and not the manipulating the hypothetical interaction in the system) in the real interacting many-body system, i.e., the interacting Fermi system (so-called the superconductor). Now, we consider concurrence in terms of the potential scattering, which was usually not considered in the investigation of the physical quantities in the SR model. At a fixed potential scattering, concurrence is enhanced by either the increase of the interaction between Cooper pair (via the energy gap) or the reduction of the absolute value of the exchange interaction. It merits mentioning that the effect of the localized excited state increases when the coupling of the impurity with conduction electrons or the exchange interaction becomes stronger (before the induced impurity band is formed). Hence, the competition of these interactions occurs in concurrence. It is worth mentioning that at the turning point (regardless of the effect of the interactions on the competition of the value of concurrence), there is the competition between the intensities of the exchange and pairing interactions which leads to the displacement of the turning point toward the lower or higher value of the potential scattering; the enhancement of the absolute value of the exchange (pair) interaction tends 
to displace the point to the higher (lower) value of potential scattering. In order to have the turning point, by the increase of the pairing interaction (or the reduction of the absolute value of the exchange interaction), the system does not need to have a large variation of the potential scattering. Another important note is that the increase of the absolute value of the exchange or the pairing interaction causes that the local maximum point occurs at the higher values of the potential scattering. It should be noted that the effect of these interactions on the value of concurrence at the local maximum point is reversed; i.e., the increase of the absolute value of the exchange (pair) interaction causes the reduction (increase) of concurrence at the local maximum point. It is worth mentioning that the increase of $|J|$ in the occurrence of QPTs always causes the increase of $V$ and the effect of $|J|$ and $V$ in concurrence is always coupled.

Concurrence versus $j$ at different fixed values of $v$, and at the fixed value of $p$ and $k_{F} r$, is depicted in Fig.5. The behavior of the curves is similar, so that by increasing the absolute value of $j$, the local maximum of the curves appears. Two regimes on the $j$ - axis are considered for investigating the curves of Fig.5. For each $j$ located in the almost interval $(-0.30,-0.60)$, by increasing the value of $v$, concurrence increases, whereas, for each $j$ located in the almost interval $(-0.60,-0.99)$, the situation is reversed. It is worth mentioning that, after lengthy but straightforward calculations with different fixed values of $p$ and $k_{F} r$, it is observed that the overview of the curves doesn't change.

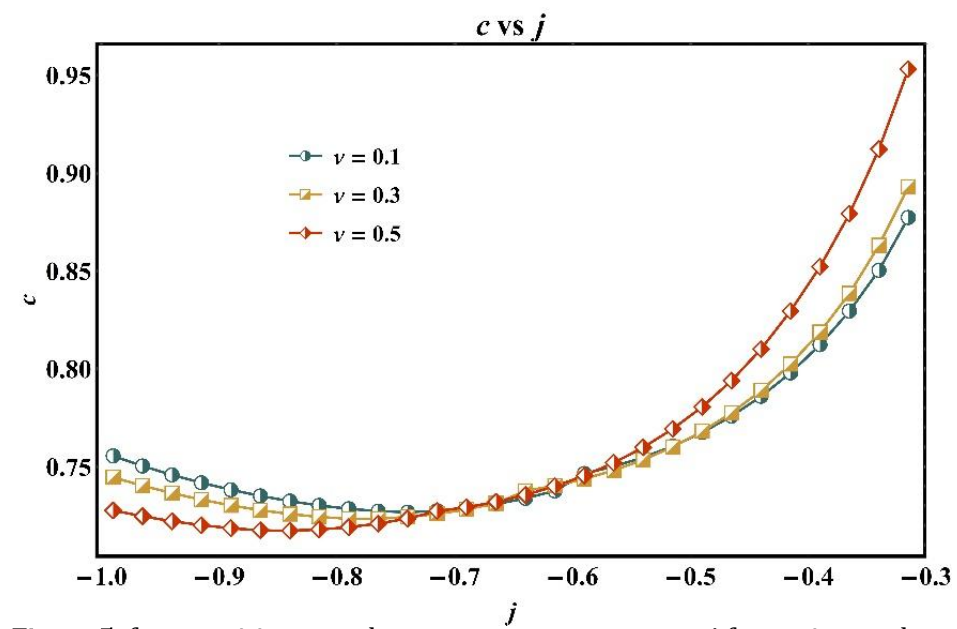

Figure 5. for transition-metal case; concurrence versus $j$ for various values of the $v$ at $p=0.2$ and $k_{F} r=1$. 
By considering a fixed value of $v(=0.1)$, a fixed value of relative distance, $r$ $\left(=1 / k_{F}\right)$, and a fixed value of the normalized impurity concentration, $p(=0.2)$, the first and second partial derivatives of concurrence versus jare depicted in Fig.6. It can be seen that at $j \approx-0.94, \partial_{j} c$ has a local minimum point and the second partial derivative is equal to zero.

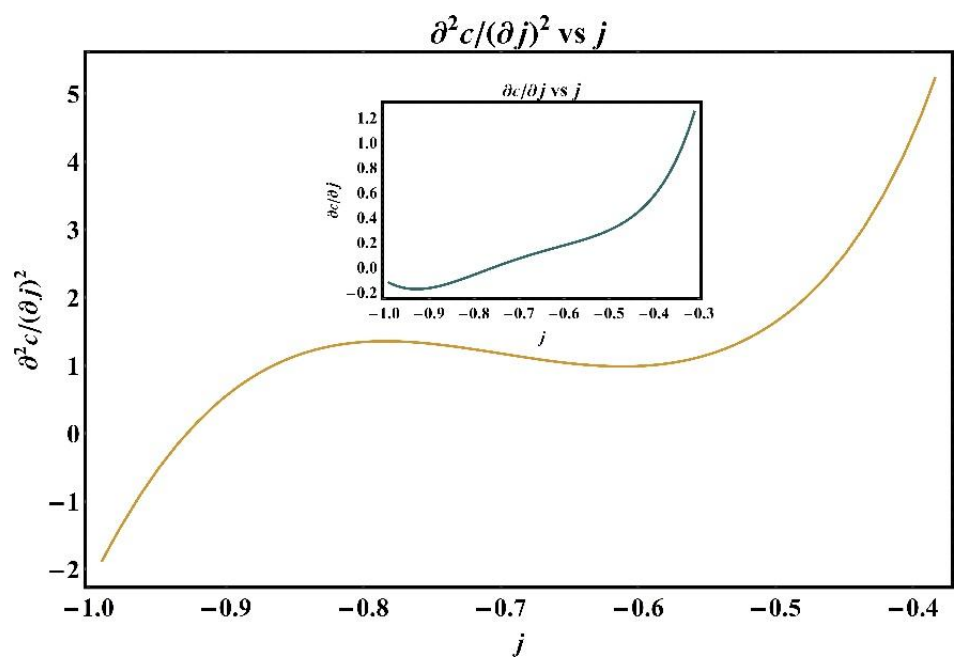

Figure 6. partial derivatives of $c$ versus $j$ at $v=0.1, k_{F} r=1$ and $p=0.2$.

As it can be seen from Figs.5 and 6, there is a turning point in the curve of $c$ versus $j$. More importantly, at a different potential scattering, the comparison of curves indicates to have different behavior on concurrence.

Concurrence versus the relative distance (of two electrons of the Cooper pair) at different fixed values of $p, v$, and $j$ is depicted in Fig.7. As it can be seen that from the overview of the figures, by increasing the value of $k_{F} r$, concurrence decreases. What's more important, at a fixed value of $k_{F} r$, the value of concurrence in the presence of the transition-metal impurity is greater than the non-impurity case. From Fig.7(a), it can be seen that the curves for different values of $v$, almost overlapped. From Fig.7(b), it can be seen that at a fixed value of $k_{F} r$, by increasing the value of $p$, concurrence increases. At the higher values of $p$, the zero value of concurrence occurs at a larger value of $k_{F} r$. Finally, from Fig.7(c), it can be seen that by increasing the absolute value of $j$, at a fixed $k_{F} r$, concurrence decreases. By considering the high value of $|j|$, the variation of the concurrence doesn't change significantly. Also, up to the first-order approximation, by 
changing the $k_{F} r$ (at fixed values of other parameters), concurrence changes, but it is not affected on the QPT points identified by $(j, v)$.
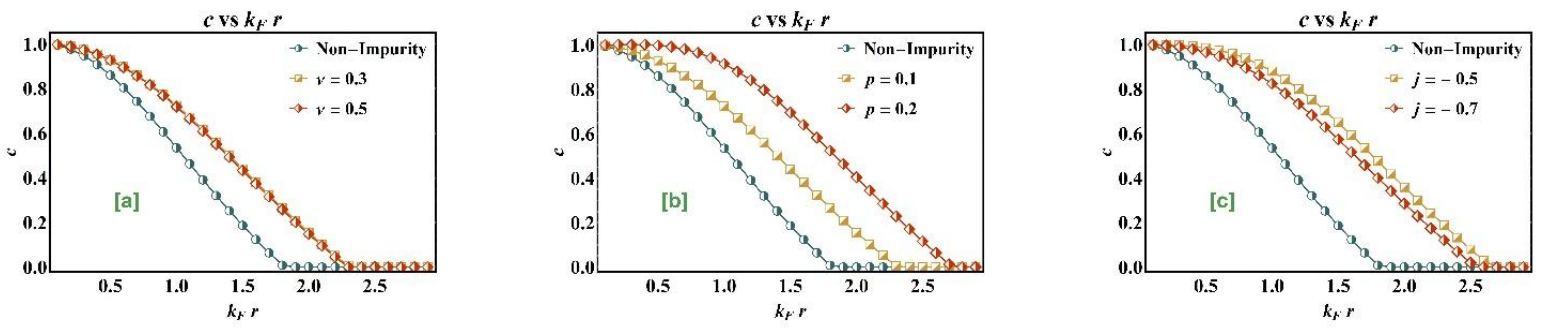

Figure 7.(a): for transition-metal case; concurrence versus $k_{F} r$ for various values of $v$ at $j=-0.8$ and $p=0.2$. (b): concurrence versus $k_{F} r$ for various values of $p$ at $j=-0.3$ and $v=0.3$. (c): concurrence versus $k_{F} r$ for various values of $j$ at $p=0.3$ and $v=0.3$.

\section{B. Non-magnetic impurity case}

We conduct comprehensive research about the effect of the non-magnetic impurities on concurrence by using the AG theory. After straightforward but lengthy calculations, Green's functions are calculated by considering the different values of $\omega_{D}$ with comparison by $\Delta$ and $\omega$. It should be mentioned that $\omega_{D}$ is finite for real materials, although, for aiming to simplify the calculations, some of authors ${ }^{21}$ supposed to have infinite value of $\omega_{D}$; chiefly, Green's functions in the presence of the impurity were considered at the infinite value of the Debye frequency, whereas, we consider both infinite and finite cases. When we use the Debye frequency with infinite (finite) value, Green's functions are so-called as $G_{N M_{1}}\left(G_{N M_{2}}, G_{N M_{3}}\right.$ and $\left.G_{N M_{4}}\right)$.

It can be seen that from the overall view of Fig. 8 (a), at a fixed collision time, $\tau(=10)$, by increasing the value of $k_{F} r$, concurrence decreases. For comparison, we bring the curve of concurrence without considering any impurity ${ }^{8,9}$. The curves of concurrence in the presence of the non-magnetic impurity with finite $\omega_{D}$ case and non-impurity case are overlapped, however, the curve of concurrence for infinite Debye frequency case is less than other cases (at a fixed value of $k_{F} r$ ). In Fig.8 (b), we focus on the investigation of concurrence versus the inverse collision time at a fixed value of $k_{F} r\left(=1 \times 10^{-3}\right)$. By increasing the value of the inverse collision time, for the infinite (finite) Debye frequency case, concurrence decreases linearly (doesn't change significantly). Concurrence in the infinite Debye frequency case is smaller than the finite Debye frequency case, at a fixed value of $1 / \tau$. 

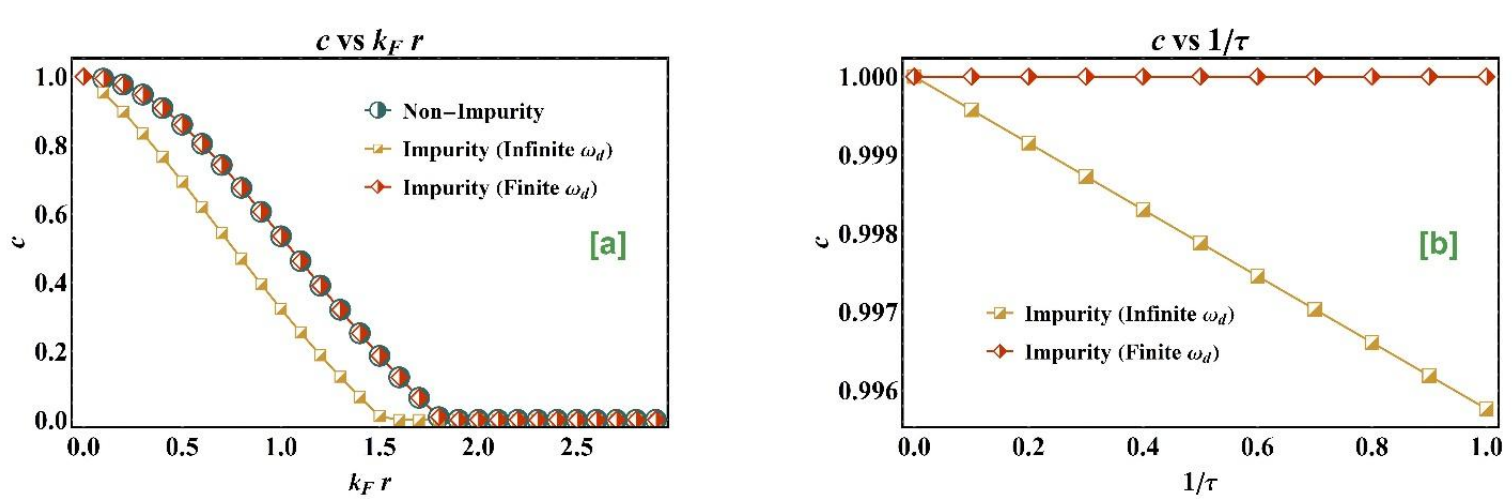

Figure 8.(a): for Rare-earth metal case; concurrence versus $k_{F} r$ for infinite and finite Debye frequency in the presence of the impurity and non-impurity cases. (b): concurrence versus inverse collision time for infinite and finite Debye frequency in the presence of the impurity and non-impurity cases.

\section{The Rare-earth metal impurity case}

The investigation of concurrence in the presence of the rare-earth impurity versus $k_{F} r, \tau_{1}$, and $\tau_{2}$ for both the infinite and finite Debye frequency cases is done. The appropriate range of the $\tau_{1}$ and $\tau_{2}$ are tuned by considering the different values of $\omega_{D}$ with comparison by $\Delta$ and $\omega$, up to the first-order approximation. After long, comprehensive and very massive calculations, we conclude that the value of the $\tau_{1}$ and $\tau_{2}$ are extraordinary large (and without physical meaning) for satisfying the approximation that we have used.

\section{Methods}

The entanglement emerges in two ways; first, due to the particle statistics, so that the non-interacting system shows entanglement, and second, due to the external or internal interaction, which is found in the few- or many-body systems, leads to the increase or decrease of the bipartite or tripartite or even multipartite entanglement ${ }^{9}$. We deal with an interacting system namely a superconductor. First, we specify the Hamiltonian model used in our work.

We begin by considering the Hamiltonian of the Bardeen-Cooper-Schrieffer (BCS) theory. The general Hamiltonian form of a s-wave superconductor ${ }^{19,22}$ is given by $H=H_{0}+H_{i m p}$, where $H_{0}$ and $H_{i m p}$ represent the effective Hamiltonian of BCS pure superconductor and the impurity part of the Hamiltonian, respectively. $H$ can be represented in terms of the field destruction operator, $\psi$, and the field creation operator, $\psi^{\dagger}$, as follows 


$$
\begin{aligned}
H_{0}=\int d^{3} x( & \sum_{\alpha} \psi_{\alpha}^{\dagger}(\vec{x})\left(\left(-i \hbar \nabla-\frac{e}{c} A\right)^{2} / 2 m-E_{F}\right) \psi_{\alpha}(\vec{x}) \\
& \left.+\sum_{\alpha} U(\vec{x}) \psi_{\alpha}^{\dagger}(\vec{x}) \psi_{\alpha}(\vec{x})+\Delta(\vec{x}) \psi_{\uparrow}^{\dagger}(\vec{x}) \psi_{\downarrow}^{\dagger}(\vec{x})+\Delta^{*}(\vec{x}) \psi_{\downarrow}(\vec{x}) \psi_{\uparrow}(\vec{x})\right)
\end{aligned}
$$

The term $U(\vec{x})$, Hartree-Fockpotential, destroys and creates one electron and therefore conserves the number of particles. On the other hand, the term $\Delta(\vec{x})$, the superconducting energy gap (order parameter), increases or decreases the number of particles by two. The physical meaning of the energy gap is minimum excitation energy and the excitation spectrum has an energy gap. The energy required to break a cooper pair is $2 \Delta$. We have

$$
\begin{aligned}
& U(\vec{x})=-U_{0}\left\langle\psi_{\uparrow}^{\dagger}(\vec{x}) \psi_{\uparrow}(\vec{x})\right\rangle=-U_{0}\left\langle\psi_{\downarrow}^{\dagger}(\vec{x}) \psi_{\downarrow}(\vec{x})\right\rangle \\
& \Delta(\vec{x})=-U_{0}\left\langle\psi_{\downarrow}(\vec{x}) \psi_{\uparrow}(\vec{x})\right\rangle=U_{0}\left\langle\psi_{\uparrow}(\vec{x}) \psi_{\downarrow}(\vec{x})\right\rangle
\end{aligned}
$$

where $U_{0}$ is a pairing interaction. Now we discuss about the spatial variations of the energy gap (for different kinds of impurities, i.e., non-magnetic, rare-earth metal, transition-metal impurities) and eventually we can conclude that its spatial variations can be neglected and the average value of the energy gap can be used; of course, in the presence of the impurity, this value has been annealed, i.e., the $\Delta$ change to $\widetilde{\Delta}$. For nonmagnetic impurities, the most important assumption underlying Anderson's theorem is that the superconducting energy gap in the presence of impurities can be taken to be uniform, $\Delta(\vec{x}) \simeq \Delta^{19-23}$. For rare-earth metal case, by considering the Abrikosov-Gorkov theory, taking into account the average value of the gap, it's proved that special variations of the superconducting gap of conventional superconductors, i.e. s-wave superconductors, is negligible because of exciting the long range of the coherence length. Furthermore, there is a small correction of the superconducting gap due to its special variations; we consider a constant superconducting gap without any errors. In fact, the effect of special variations of the superconducting gap in the vicinity of the impurity maybe significant in the superconductors with short coherence length such as high $-T_{c}$ cuprate superconductors, which is not under investigation in this work ${ }^{44-48}$. For transition-metal case, by considering the Shiba-Rusinov model, when an impurity is induced into a superconductor, $\Delta(\vec{x})$ is expected to experience local changes. Of course, this change depends on the strength of the impurity potential. However, local variations of 
$\Delta(\vec{x})$ rapidly decrease away from an impurity center. Therefore, we anticipate that the superconducting energy gap, $\Delta(\vec{x})$, is a self-averaging quantity, at least at low enough impurity concentrations. It will be seen from the results below that the assumption $\Delta(\vec{x}) \simeq \Delta$ indeed turns out to bevalid. The exact calculation of the scattering of an electron by an isolated magnetic impurity leads to the appearance of local impurity levels inside the energy gap, corresponding to excited states of Cooper pairs near the impurity. It should be mention that we neglect the local variation of the order parameter around an impurity spin. Although, this approximation is employed also by some authors. To neglect the local variation of $\Delta(\vec{x})$ may be allowed when one deal with thermodynamic properties of a superconductor with paramagnetic impurities, but, it may not be allowed when we calculate quantities that strongly reflect local properties around a paramagnetic impurity $^{25-27}$. Eventually, it merits mentioning that the $\Delta(\vec{x})$ has been replaced by $\Delta$ for these different kinds of impurities that used in this work.

The impurity part of the Hamiltonian consists of the interaction scattering term due to the $V(\vec{x})$, which is independent of spin and the exchange interaction term. If the impurity atom has a magnetic moment, there is an exchange interaction between the local spin on the impurity site and the conduction electrons; the intensity of the exchange interaction is given by $J(\vec{x})$. Then, we have

$$
H_{i m p}=\sum_{\alpha \beta} \int d^{3} x J(\vec{x}) \psi_{\alpha}^{\dagger}(\vec{x}) S . \sigma_{\alpha \beta} \psi_{\beta}(\vec{x})+\int d^{3} x \sum_{\alpha} \psi_{\alpha}^{\dagger}(\vec{x}) V(\vec{x}) \psi_{\alpha}(\vec{x})
$$

where $\alpha$ and $\beta$ are spin indices. $\psi$, and $\psi^{\dagger}$, respectively, are the fermionic field creation and annihilation field operators. The range of the exchange interaction is determined by the quantum mechanical structure of the electron cloud associated with the localized spin. Indeed, $V(\vec{x})$ and $J(\vec{x})$ are the impurity's non-magnetic and magnetic scattering potential, respectively. The term $V(\vec{x})$ explains an arbitrary external potential that describes the effects of impurities and of the specimen surface. $J(\vec{x})$ is the exchange interaction and appears between conduction electrons and single impurities and here assumed $J_{0} \delta(\vec{x}-$ $\overrightarrow{x_{0}}$ ), which captures the essential physics of the problem. With this description, the exchange part in momentum space has a momentum-independent value. The $\mathrm{S}$ is the spin operator of the impurity with spin $\mathrm{S}$ and $\sigma$ are the Pauli matrices.

Furthermore, $H_{0}$ is given by 


$$
H_{0}=\sum_{\vec{k} \alpha} \epsilon_{\vec{k}} c_{\vec{k} \alpha}^{\dagger} c_{\vec{k} \alpha}+\sum_{\vec{k}} \Delta_{\vec{k}}\left(c_{\vec{k} \uparrow}^{\dagger} c_{-\vec{k} \downarrow}^{\dagger}+c_{-\vec{k} \downarrow} c_{\vec{k} \uparrow}\right)
$$

where $\vec{k}$ is the wave vector, $\epsilon_{\vec{k}}$ is the kinetic energy with respect to chemical potential, $c_{\vec{k} \alpha}^{\dagger}$ and $c_{\vec{k} \alpha}$ represent creation operator and annihilation operator of spin $\alpha$, respectively. For s-wave superconductor, $\Delta_{\vec{k}}$ isn't dependent on momentum.

Also, the impurity part of the Hamiltonian can be written as

$$
H_{i m p}=\frac{1}{2 N} \sum_{k, k^{\prime}, \alpha, \beta} J\left(\vec{k}-\vec{k}^{\prime}\right) c_{\vec{k}, \alpha}^{\dagger} \sigma_{\alpha \beta} \cdot S c_{\vec{k}^{\prime}, \beta}+\sum_{\vec{k}, \vec{k}^{\prime}, \alpha} V c_{\vec{k} \alpha}^{\dagger} c_{\vec{k}^{\prime} \alpha}
$$

Basically, the exchange part of $H_{i m p}$ was written in terms of the quantities and operators in momentum space. For a specific host and specific impurity, we suppose a constant value for the intensity of the exchange interaction, $J\left(\vec{k}-\vec{k}^{\prime}\right)$. We deal with two kinds of elements that we use as impurities, i.e., transition-metal impurities and rare-earth impurities. For transition-metal case, we consider scattering on classical spins that first studied independently at about the same time by Shiba, Rusinov, and Yu. Quantum mechanical properties of spin can be neglected when $S \rightarrow \infty$, and we simultaneously take $J \rightarrow 0$, so that the product $J S$ have a constant value. Basically, the localized spin acts as a local magnetic field. In contrast to the transition-metal case, for the rare-earth metal case, the quantum mechanical property of spins is used and eventually appeared on the collision times ${ }^{20-21,24,41}$.

The concurrence and entanglement can be calculated by the reduced spin-space density matrix arrays and Green's functions. Since our system under investigation is an interacting Fermi gas, we consider the trick that causes the procedure calculating the concurrence in the non-interacting systems can be used for the interacting systems. Previously, for s-wave and d-wave superconductors as the interacting systems, the trick has been used and the only change was to use Green's functions for superconductors instead of non-interacting Fermi gases ${ }^{8-9,13}$. The reason for using the formulation of the interacting system based on the non-interacting system can be explained as follows; first, a superconducting ground state can be written as a product of the ground state of a noninteracting Fermi system to an extended Jastrow function or Feenberg factor ${ }^{9,42-43}$. The factor is related to the interactions and also, bipartite entanglement in the non-interacting part of the state can be modified by Feenberg factor. Second, by using the Bogoliubov 
transformation, the Hamiltonian of an interacting system can be diagonalized as a noninteracting system but with different energy ${ }^{9}$. Of course, the existence of impurity in superconductors will cause to product an additional interaction, which can be considered as a small perturbation on superconductors, accompanied by new Green's functions. Thereby, the formulation of the concurrence in terms of the non-interacting Green's functions can be survived. It should be mentioned that for both transition-metal and rareearth metal cases we will see that the new Green's functions (in the presence of the impurity) have the same form as the absence of the impurity case, but only by transforming the frequency to a new one. Another important point is about the structure of the BCS theory, in which mean-field approximation is used. We know that the entanglement can be changed by this approximation so that, the results of bipartite entanglement maybe incorrect unless the fluctuation related to all interactions can be considered to be small (in throughout the paper, the smallness of the fluctuation is considered).

By these assumptions and explanation, first, we bring the relation between reduced spin-space density matrix (elements are written in the spin space and each of them has position-dependence) and Green's functions (fermionic case) of a superconductor. The relation between the two-electron space-spin density matrix and the two-particle Green's function is described by

$$
\rho_{2}\left(\vec{x}_{1}, \vec{x}_{2}, \vec{x}_{1}^{\prime}, \vec{x}_{2}^{\prime}\right)=(-1 / 2) G\left(\vec{x}_{1} t_{1}, \vec{x}_{2} t_{2} ; \vec{x}_{1}^{\prime} t_{1}^{+}, \vec{x}_{2}^{\prime} t_{2}^{+}\right)
$$

where $t_{i}^{+}$denotes time infinitesimally later than $t$. The two-particle Green's function for the superconducting state can be written in terms of single-particle Green's functions ${ }^{8}$ as follows

$$
\begin{aligned}
\rho_{s_{1}, s_{2} ; s_{1}^{\prime}, s_{2}^{\prime}}\left(\vec{x}_{1}, \vec{x}_{2}, \vec{x}_{1}^{\prime}, \vec{x}_{2}^{\prime}\right) \\
=(-1 / 2)\left(G_{s_{1} s_{1}^{\prime}}\left(\vec{x}_{1} t_{1}, \vec{x}_{1}^{\prime} t_{1}^{+}\right) G_{s_{2} s_{2}^{\prime}}\left(\vec{x}_{2} t_{2}, \vec{x}_{2}^{\prime} t_{2}^{+}\right)\right. \\
\left.-G_{s_{1} s_{2}^{\prime}}\left(\vec{x}_{1} t_{1}, \vec{x}_{2}^{\prime} t_{2}^{+}\right) G_{s_{2} s_{1}^{\prime}}\left(\vec{x}_{2} t_{2}, \vec{x}_{1}^{\prime} t_{1}^{+}\right)\right)
\end{aligned}
$$

where $s_{1}, s_{2}, s_{1}^{\prime}$ and $s_{2}^{\prime}$ are particles' spin indices. The single particle Green's function $G_{\alpha \beta}\left(\vec{x} t, \vec{x}^{\prime} t^{\prime}\right)$ is defined by ${ }^{2,22}$

$$
i G_{\alpha \beta}\left(\vec{x} t, \vec{x}^{\prime} t^{\prime}\right)=\frac{\left\langle\psi_{0}\left|T\left[\hat{\psi}_{H \alpha}(\vec{x} t) \hat{\psi}_{H \beta}^{\dagger}\left(\vec{x}^{\prime} t^{\prime}\right)\right]\right| \psi_{0}\right\rangle}{\left\langle\psi_{0} \mid \psi_{0}\right\rangle}
$$

where $\left|\psi_{0}\right\rangle$ is Heisenberg ground state. As an instance $\widehat{\psi}_{H \alpha}(\vec{x} t)$ is a Heisenberg operator 
with the time dependence and describes by $\hat{\psi}_{H \alpha}(\vec{x} t)=e^{i \widehat{H} t / \hbar} \hat{\psi}_{\alpha}(\vec{x}) e^{-i \widehat{H} t / \hbar}$. The field operators, $\hat{\psi}_{\alpha}(\vec{x})$ and $\hat{\psi}_{\beta}^{\dagger}\left(\vec{x}^{\prime}\right)$, satisfy anti-commutation relations ${ }^{2,22}$

$$
\begin{aligned}
& \left\{\hat{\psi}_{\alpha}(\vec{x}), \hat{\psi}_{\beta}^{\dagger}\left(\vec{x}^{\prime}\right)\right\}=\delta_{\alpha \beta} \delta\left(\vec{x}-\vec{x}^{\prime}\right) \\
& \left\{\hat{\psi}_{\alpha}(\vec{x}), \hat{\psi}_{\beta}\left(\overrightarrow{x^{\prime}}\right)\right\}=\left\{\hat{\psi}_{\alpha}^{\dagger}(\vec{x}), \hat{\psi}_{\beta}^{\dagger}\left(\overrightarrow{x^{\prime}}\right)\right\}=0
\end{aligned}
$$

It should merit mentioning that we have ignored the anomalous Green's function of the system in Eq. (7), because, the unperturbed anomalous Green's function in comparison to the single-particle Green's function can be neglected. According to that, the small modification due to the impurity on anomalous Green's function also is so small. And eventually, the normalized reduced space-spin density matrix in the presence of the impurity (for both transition-metal and rare-earth metal cases) is written as follows $^{8-9,49}$

$$
\tilde{\rho} \equiv \frac{\rho}{\operatorname{Tr}[\rho]}=(1-\Lambda) \frac{\mathbf{I}}{4}+\Lambda\left|\psi^{(-)}\right\rangle\left\langle\psi^{(-)}\right|
$$

where I is a $4 \times 4$ unit matrix and $\left|\psi^{(-)}\right\rangle=\frac{1}{\sqrt{2}}(|\uparrow \downarrow\rangle-|\downarrow \uparrow\rangle)$ is the singlet state. It should be mentioned that the density matrix is the Werner state (as the pure conventional superconductor case). By considering the case $\vec{x}_{1}=\vec{x}_{1}^{\prime}, \vec{x}_{2}=\vec{x}_{2}^{\prime}$ and $\vec{r}=\vec{x}_{1}-\vec{x}_{2}$, the parameter $\Lambda$ is obtained by calculating the reduced density matrix $\tilde{\rho}$ and given by a function of $G(r)$ as follows 9

$$
\Lambda=G(r) G(-r) /(2-G(r) G(-r))
$$

The $G(r)$ specified by $G_{s_{1} s_{2}^{\prime}}\left(\vec{x}_{1} t_{1}, \vec{x}_{2}^{\prime} t_{1}^{+}\right) \equiv \delta_{s_{1} s_{2}^{\prime}} G(r)$ where $r$ is absolute value of $\vec{r}$ vector. The $\Lambda$, up to the first order approximation, is obtained as

$$
\Lambda \approx \Lambda^{(0)}+\frac{4\left[g^{(0) 2}(r) d(r)+q(r)\right]}{G^{(0) 2}(0)\left[2-g^{(0) 2}(r)\right]^{2}}
$$

where $\Lambda^{(0)}$ is non-impurity part of the $\Lambda$ and we have

$$
\begin{aligned}
& g^{(0)}(r)=G^{(0)}(r) / G^{(0)}(0) \\
& d(r)=\operatorname{Im}\left[G^{(1)}(0)\right] \times \operatorname{Im}\left[G^{(0)}(0)\right] \\
& q(r)=-2 \operatorname{Im}\left[G^{(1)}(r)\right] G^{(0)}(r)
\end{aligned}
$$

It should be noted that Eq.(12) is different than that given in Ref.[49] (here $\Lambda$ was written in terms of $\left.r\left(\equiv\left|\vec{x}_{1}-\vec{x}_{2}\right|\right)\right)$. The Green's functions without impurities (unperturbed Green's functions) is given by $G^{(0)}(r)$ and the first approximation of the 
Green's functions due to the presence of impurities is represented by $G^{(1)}(r)$. The concurrence in terms of $\Lambda$, as a measure of quantum bipartite entanglement ${ }^{3,14}$, is given by

$$
C=\max \{0,(3 \Lambda-1) / 2\}
$$

The intended Green's functions for calculating the $\Lambda$ and subsequently concurrence will be obtained for rare-earth and transition-metal cases separately. For both of them, Green's functions in position space will be used. Basically, Green's function is the results of Fourier transform $G(r, t)$ of a function $G(\mathrm{k}, \omega)$.

Transition-metal case. We consider the transition-metal impurity as a perturbation in Green's functions and its effect on the reduced density matrix and eventually on the concurrence of the system. The exact Green's functions in the presence of the impurity is described by ${ }^{25-28,55}$

$$
\bar{G}_{\vec{k}}=\left(a_{1}+a_{2} \rho_{2} \sigma_{2}+a_{3} \rho_{3}\right)
$$

where $a_{n(=1,2,3)}=\tilde{b}_{n} /\left(\widetilde{\omega}^{2}-\tilde{\Delta}^{2}-\tilde{\epsilon}_{\vec{k}}^{2}\right), \tilde{b}_{1}=\widetilde{\omega}, \tilde{b}_{2}=\tilde{\Delta}$ and $\tilde{b}_{3}=\tilde{\epsilon}_{\vec{k}}$. Also, $\widetilde{\omega}, \tilde{\epsilon}_{\vec{k}}$, and $\tilde{\Delta}$ are the renormalized frequency, renormalized kinetic energy with respect to chemical potential, and renormalized order parameter, respectively. It should be mentioned that $\tilde{b}_{n}$ is represented by

$$
\tilde{b}_{n}=b_{n}+\left(\Gamma_{n} \times \zeta_{n}\left(j, v, U\left(\tau_{s}, \omega / \Delta, j, v\right)\right)\right) ; \Gamma_{n}=p t_{n}(j, v) / 16 \gamma j^{2} \pi N(0)
$$

where $b_{1}, b_{2}$ and $b_{3}$ are $\omega, \epsilon_{\vec{k}}$ and $\Delta$, respectively. The $\zeta_{n}$ is expressed by the composition of $U$ and $\epsilon_{0}$ as follows

$$
\zeta_{1}\left(j, v, U\left(\tau_{s}, \omega / \Delta, j, v\right)\right)=\frac{U\left(1-U^{2}\right)^{\frac{1}{2}}}{\left(\epsilon_{0}^{2}-U^{2}\right)} ; \zeta_{2,3}\left(j, v, U\left(\tau_{s}, \omega / \Delta, j, v\right)\right)=\frac{\left(1-U^{2}\right)^{1 / 2}}{\left(\epsilon_{0}^{2}-U^{2}\right)}
$$

where $\epsilon_{0}=\left(1+v^{2}-j^{2}\right)\left(\left(1+v^{2}-j^{2}\right)^{2}+4 j^{2}\right)^{-1 / 2}$ and $U$ has the following from

$$
\omega / \Delta=U\left[1-\alpha\left(1-U^{2}\right)^{\frac{1}{2}} /\left(\epsilon_{0}{ }^{2}-U^{2}\right)\right], \quad \alpha=p / 8 \Delta \gamma \pi N(0)
$$

where $p$ is normalized impurity concentration ${ }^{30-33}, \ln \gamma$ is Euler's constant, the $N(0)$ is the density of single-particle states at the Fermi level in the normal metal. The $\Gamma_{n}, \epsilon_{0}$, and $\alpha$ (or, equivalently, $j, v$, and $p$ ) show the impurity effect on Green's functions. The values 
of the $\Gamma_{n}$ and $\alpha$ are small. Although, basically, $\alpha$ also consists of $\Gamma_{n}$ s. It should be mentioned that the multiplication relation of $\Gamma_{n}$ and $\alpha$ appear in the Green's functions and by considering this reality that we investigate the first-order approximation with respect to the impurity effect on Green's functions, so we must neglect the terms like the $\Gamma_{n}{ }^{2}$ (or $\left.\Gamma_{n} \times \alpha\right)$. For this reason, we must ignore all orders of the $\alpha$ (because, the first order of $\Gamma_{n}$ cannot be eliminated). It should be merits mentioning that the value of the $\alpha$ can be controlled by using the normalized impurity concentration. So we assume that $U \approx \omega / \Delta$. The $t_{n}(j, v)$ is given by

$$
t_{1}(j, v)=\left(v^{2}-j^{2}\right)^{2}+j^{2}+v^{2}, t_{2}(j, v)=v\left(1+v^{2}-j^{2}\right), t_{3}(j, v)=t_{1}(j, v)-2 j^{2}
$$

where $j$ and $v$ are defined by the strength of the exchange interaction and the potential scattering, respectively. As we said that, the exchange interaction appears between conduction electrons and single impurities and undoubtedly the exchange interaction has different values for different kinds of impurities and hosts ${ }^{50}$. We consider the kinds of the magnetic and non-magnetic impurities and this means that, we have both the potential scattering and exchange interaction. However, in some researches, the potential scattering has been ignored and the exchange interaction was investigated by using thermodynamics properties, transport properties and magnetic properties ${ }^{51-52}$.

Basically, the low value of the perturbed Green's functions in comparison to the primary Green's function allows us for using the first-order approximation. This reality leads to the results about choosing the allowed value for the exchange interactions, potential scattering and normalized impurity concentration. Therefore, the potential scattering and exchange interaction cannot be separately obtained so, the particular limitation of the exchange interaction affects on the allowed limitation of the potential scattering and additionally, the normalized impurity concentration is another parameter that influences on concurrence. Therefore, we must examine the occurrence of these limitations simultaneously. We address the investigation of this issue in two different aspects, the numerical aspect and the experimental aspect. We will clearly identify that why we choose the specific limitation of parameters that are not chosen, arbitrarily. Before investigating these two aspect, we clear the general range of the $p, v$ and $j$. This is obvious that the low value of the normalized impurity concentration is acceptable. Also, one may note that $\left|\epsilon_{0}\right|<1$, by using this condition the allowed range of potential scattering is $(0,1)$ and the allowed range of the exchange interaction is $(-1,0)$. First, we 
start with the numerical results. For reducing the complexity of the numerical calculations, we consider some hypotheses, such as the smallness of the perturbed Green's

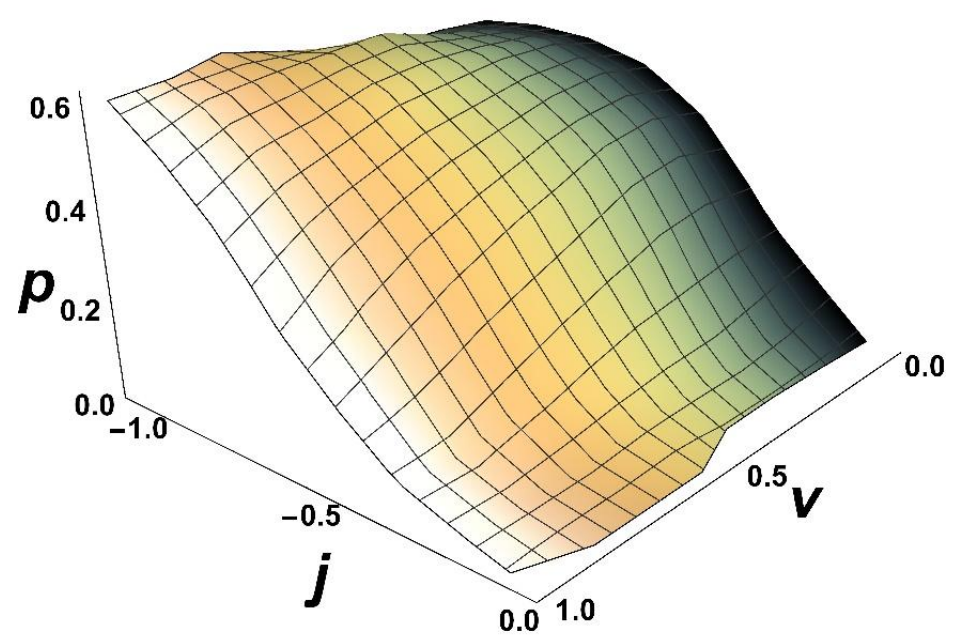

Figure 9. The permissible range (under the manifold) of $j, v$ and $p$ with respect to the low value of the $\operatorname{Im}\left[G^{(1)}(r)\right] / \operatorname{Im}\left[G^{(0)}(0)\right]$

functions in comparison with the unperturbed Green's functions and the smallness of the appeared parts due to the impurities in the renormalized order parameter (or energy gap), and the single-particle energy and the frequency. The numerical results clearly show that the exchange interaction, potential scattering and normalized impurity concentration are extremely dependence together. So that, for choosing each value of them, we must investigate the limitation of other parameters. By using our numerical results, we obtain that the allowed range of the parameters are $p<0.6, j<-0.2$ and $0 \lesssim v \lesssim 1$. Again, it should be mentioned that the chosen values are dependent together that exactly, the situation of the parameters in comparison together is obtained in Fig. 9.

After observing the numerical results, we address the investigation of these parameters by using the experimental evidences via the physical importance aspect (as an instance $\epsilon_{0}$ was calculated under the thermodynamic properties ${ }^{53-54}$ ). The investigation of the experimental results shows that the low values of the normalized impurity concentration are acceptable and also, by considering the $\epsilon_{0}$ for some special cases like $\mathbf{I n}-\mathrm{Fe}^{53}, \mathbf{P b}-\mathrm{Mn}^{53}$ and $\mathbf{Z n}-\mathrm{Mn}^{54}$, we obtain the appropriate range of the exchange interaction and potential scattering, which is represented by bellow data sheet: 


\begin{tabular}{|c|c|c|c|}
\hline sample & $\boldsymbol{\epsilon}_{\mathbf{0}}$ & $\boldsymbol{j}$ & $\boldsymbol{v}$ \\
\hline In-Fe & 0.60 & $-0.85<j<-0.50$ & $0<v<1$ \\
\hline Pb-Mn & 0.55 & $-0.90<j<-0.54$ & $0<v<1$ \\
\hline Sample A & 0.45 & $-0.99<j<-0.61$ & $0<v<1$ \\
\hline Sample B & 0.35 & $-1 .<j<-0.69$ & $0 .<v<0.86$ \\
\hline Zn-Mn & 0.25 & $-1<j<-0.77$ & $0<v<0.71$ \\
\hline
\end{tabular}

In the above data sheet, Sample A and B have chosen typically. In each sample, by considering a certain value of the exchange interaction in the allowed range, we must choose only a certain value of the potential scattering. Eventually, the conclusion of the numerical and experimental investigations shows that the low value of the normalized impurity concentrations is acceptable, subsequently, the absolute value of the exchange interaction, which is placed in the high value of the exchange interaction range, is acceptable and eventually, the whole range of the potential scattering is acceptable, roughly. Here, it should be mentioned that the relation between these three parameters is extraordinarily complicated and in each part of the work, we examine the situation of them. With these descriptions, we obtain that $t_{n}(j, v)$ s are always less than one.

Basically, the Green's functions in the presence of impurity scattering are given by

$$
G_{\vec{k} \overrightarrow{k^{\prime}}}=G_{\vec{k}}^{0} \delta_{\vec{k} \overrightarrow{k^{\prime}}}+G_{\vec{k}}^{0} T G \underset{k^{\prime}}{0}
$$

where the scattering is described by a $T-$ matrix $x^{55}$ and the general form of the $G_{\vec{k}}^{0}$ is a tensor product of Pauli-spin matrices and electron-hole spin states $\left(G_{\vec{k}}^{0}=\left(\omega-\epsilon_{\vec{k}} \rho_{3}-\right.\right.$ $\left.\Delta \rho_{2} \sigma_{2}\right)^{-1}$ ). The first array of this matrix is given by

$$
G^{(0)}(\vec{k}, \omega)=\frac{\omega+\epsilon_{\vec{k}}}{\omega^{2}-\Delta^{2}-\epsilon_{\vec{k}}^{2}}
$$

By considering this reality that the value of the $\alpha$ is low and as discussed above, up to the first approximation, we neglect the part of $T$-matrix that depended on the $\alpha$. The first term of the series expansion for $T$ - matrix about $\alpha=0$ is obtained by $T^{(0)}=$ $\left(S_{1} \Gamma_{1}+S_{2} \Gamma_{2} \rho_{2} \sigma_{2}+S_{3} \Gamma_{3} \rho_{3}\right)$ where $S_{1}, S_{2}$ and $S_{3}$ are

$$
S_{1}=\frac{-\omega \sqrt{\Delta^{2}-\omega^{2}}}{\left(\Delta^{2} \epsilon_{0}^{2}-\omega^{2}\right)}, \quad S_{2}=\frac{\Delta \sqrt{\Delta^{2}-\omega^{2}}}{\left(\Delta^{2} \epsilon_{0}^{2}-\omega^{2}\right)}, \quad S_{3}=\frac{\Delta^{2}-\omega^{2}}{\left(\Delta^{2} \epsilon_{0}^{2}-\omega^{2}\right)}
$$

Eventually, the first array of the Green's function matrices is obtained as follows 


$$
G(\vec{k}, \omega)=\frac{\omega+\epsilon_{\vec{k}}}{\omega^{2}-\Delta^{2}-\epsilon_{\vec{k}}^{2}}+O_{1} \Gamma_{1}-O_{2} \Gamma_{2}+O_{3} \Gamma_{3}
$$

where three last terms of Eq.(23) represent the perturbed Green's function and we obtain the first order approximation of the Green's function as follows

$$
\begin{aligned}
G^{(1)}(\vec{k}, \omega)= & \Xi(\vec{k}, \omega)\left(\Gamma_{1} \omega\left(\left(\omega+\epsilon_{\vec{k}}\right)^{2}+\Delta^{2}\right)-2 \Gamma_{2} \Delta^{2}\left(\omega+\epsilon_{\vec{k}}\right)\right. \\
& \left.-\Gamma_{3}\left(\Delta^{2}-\omega^{2}\right)^{1 / 2}\left(\left(\omega+\epsilon_{\vec{k}}\right)^{2}-\Delta^{2}\right)\right)
\end{aligned}
$$

where

$$
\Xi(\vec{k}, \omega)=\frac{\left(\Delta^{2}-\omega^{2}\right)^{1 / 2}}{\left(\omega^{2}-\Delta^{2}-\epsilon_{\vec{k}}^{2}\right)^{2}\left(\omega^{2}-\Delta^{2} \epsilon_{0}^{2}\right)}
$$

First, by doing the integral of $G(\vec{k}, \omega)$ with respect to thewon the interval, $(\Delta,-\Delta)$, and then, by rewriting $k$ as $k=k_{F}+\epsilon_{\vec{k}} r / v_{F}$ in the Green's function and eventually, the numerical approximation to the integral of $G(\vec{k})$ with respect to the $\epsilon_{\vec{k}}$ on the interval, $\left(\omega_{D},-\omega_{D}\right)$, the perturbed Green's function at position space is obtained.

According to the numerical calculation for transition-metal case, it is seen that the effect of the Debye frequency variation is insignificant, so, we choose $0.1 \mathrm{eV}$ as the typical value of the Debye frequency. The value of $\Delta$ is measured with respect to the Fermi energy and we choose $0.001 \mathrm{eV}$ as the typical value of that (of course, we have investigated the different values of the order parameter corresponding to the different hosts). Eventually the $G(r)$ is given by

$$
G(r)=\frac{(2 \pi)^{5 / 2} N(0)}{r p_{F}} \int_{-\omega_{D}}^{\omega_{D}} d \epsilon_{\vec{k}} \int_{-\Delta}^{\Delta} d \omega G\left(\epsilon_{\vec{k}}, \omega\right) \sin \left(p_{F} r+\epsilon_{\vec{k}} r / v_{F}\right)
$$

Rare-earth metal impurity and nonmagnetic impurity cases. We investigate the impurity effect on the BCS superconductors via concurrence. It is supposed that there are no correlations between different impurities. Usually, the existing Green's functions are defined in momentum space and frequency space, in spite of that, we need to the definition of them in position space. The Fourier transform is an appropriate tool for changing the basis. The Green's functions in position space are obtained by the analytical and numerical forms of their Fourier transform. We have been trying to change the basis, 
analytically, as much as possible but because of the complexity of equations, we had to use the numerical form of the Fourier transform in some part of this process.

The most important question is the limitation in choosing of the Debye Frequency. In fact, in the previous researches, the different conditions of the Debye frequency were not considered. Because, we would like to have a full discussion, we try to find an appropriate solution for the different conditions of the Debye frequency. We classify the finite and infinite range of Debye Frequency. Although, it merits mentioning that by considering these conditions, the changing of the basis, analytically, is extraordinarily difficult and needs the numerical work. In each circumstance of the Debye frequency, by considering the smallness of the impurity part of the Green's function we can obtain the Green's function up to the first-order approximation as the transition metal case.

The solution of the Abrikosov-Gorkov equations was used for rare-earth metal impurities and non-magnetic impurities. The Abrikosov-Gorkov equations at non-zero temperature are given by ${ }^{21}$

$$
\begin{gathered}
\left(i \omega_{n}-\epsilon_{\vec{k}}-\bar{G}\left(\omega_{n}\right)\right) G\left(\vec{k}, \omega_{n}\right)+\left(\Delta+\bar{F}\left(\omega_{n}\right)\right) F^{\dagger}\left(\vec{k}, \omega_{n}\right)=1 \\
\left(i \omega_{n}+\epsilon_{\vec{k}}+\bar{G}\left(-\omega_{n}\right)\right) F^{\dagger}\left(\vec{k}, \omega_{n}\right)+\left(\Delta^{*}+\bar{F}^{\dagger}\left(\omega_{n}\right)\right) G\left(\vec{k}, \omega_{n}\right)=0
\end{gathered}
$$

where the effect of the impurities appears in $\bar{G}\left(\omega_{n}\right), \bar{F}^{\dagger}\left(\omega_{n}\right)$ and $\bar{F}\left(\omega_{n}\right)$ terms and we have

$$
\bar{G}\left(\omega_{n}\right)=\frac{n}{(2 \pi)^{3}} \int\left|U\left(\vec{k}-\overrightarrow{k^{\prime}}\right)\right|^{2} G\left(\overrightarrow{k^{\prime}}, \omega_{n}\right) d^{3} k^{\prime}
$$

$$
\left.\bar{F}^{\dagger}\left(\omega_{n}\right)=\frac{n}{(2 \pi)^{3}} \int\left|U\left(\vec{k}-\overrightarrow{k^{\prime}}\right)\right|^{2} F^{\dagger}\left(\overrightarrow{k^{\prime}}, \omega_{n}\right) d^{3} k^{\prime} \quad \text { (and similarly for } \bar{F}\left(\omega_{n}\right)\right) \text {. }
$$

where $U\left(\vec{k}-\overrightarrow{k^{\prime}}\right)$ represents the non-magnetic part of the potential. The property $\bar{G}\left(-\omega_{n}\right)=-\bar{G}\left(\omega_{n}\right)$ was used. In the absence of external electromagnetic fields and currents, the $\Delta$ can be chosen to be real. We address the Green's functions at zero temperature by taking into account the transformation $\omega_{n} \rightarrow-i \omega$ and eventually, the solution of the above equations is given by

$$
G(\vec{k}, \omega)=\frac{\left(\widetilde{\omega}+\epsilon_{\vec{k}}\right)}{\left(\widetilde{\omega}^{2}-\epsilon_{\vec{k}}^{2}-|\tilde{\Delta}(\omega)|^{2}\right)}
$$




$$
F^{\dagger}(\vec{k}, \omega)=\frac{-\tilde{\Delta}(\omega)}{\left(\widetilde{\omega}^{2}-\epsilon_{\vec{k}}^{2}-|\tilde{\Delta}(\omega)|^{2}\right)}
$$

with the notations

$$
\widetilde{\omega}=\omega-\bar{G}(\omega) ; \quad \tilde{\Delta}(\omega)=\Delta+\bar{F}^{\dagger}(\omega)
$$

The integration over $\epsilon_{\vec{k}}$ in Eq.(28) at the symmetrical interval of $\epsilon_{\vec{k}}$ leads to the

$$
\bar{G}(\omega) / \widetilde{\omega}=-\bar{F}^{\dagger}(\omega) / \Delta
$$

and subsequently the above equation also leads to the following $\widetilde{\omega}$ and $\tilde{\Delta}$ (these quantities appear in the Green's functions in the presence of the impurity)

$$
\widetilde{\omega}=\omega \eta_{\omega}, \quad \tilde{\Delta}=\Delta \eta_{\omega}
$$

where $\eta_{\omega}$ given by

$$
\eta_{\omega}=1-\frac{1}{2 \pi \tau} \int_{-\omega_{d}}^{\omega_{d}} \frac{d \epsilon_{\vec{k}}}{\omega^{2}-\Delta^{2}-\epsilon_{\vec{k}}^{2}}
$$

The above form of $\eta_{\omega}$ used for non-magnetic impurity case and the collision time, $\tau$, is given $b^{21}$,

$$
\frac{1}{\tau}=\frac{n m p_{F}}{(2 \pi)^{2}} \int|U(\theta)|^{2} \mathrm{~d} \Omega
$$

It merits mentioning that for rare-earth metal impurities, the $\bar{G}$ and $\bar{F}^{\dagger}$ consist of the magnetic part of the potential and the above process repeats for rare-earth metal impurities, which is a kind of the magnetic impurities. The only difference is the existence of the $\eta_{\omega_{1}}\left(\widetilde{\omega}=\omega \eta_{\omega_{1}}\right)$ and $\eta_{\omega_{2}}\left(\tilde{\Delta}=\Delta \eta_{\omega_{2}}\right)$ that consist of $\tau_{1}$ and $\tau_{2}$, respectively. The definitions of $\tau_{1}$ and $\tau_{2}$ are as follows

$$
1 / \tau_{i} \propto V^{2} \pm S(S+1) J^{2} / 4 ; i=1,2
$$

The $\eta_{\omega}$ in two general separate conditions, i.e., $\omega^{2}>\Delta^{2}$ and $\omega^{2}<\Delta^{2}$ is given as follows:

$$
\begin{array}{ll}
\eta_{\omega}=1+\frac{1}{\pi \tau} \frac{\operatorname{ArcTan}\left[\frac{\omega_{D}}{\sqrt{\Delta^{2}-\omega^{2}}}\right]}{\sqrt{\Delta^{2}-\omega^{2}}}, & \Delta^{2}>\omega^{2} \\
\eta_{\omega}=1-\frac{1}{\pi \tau} \frac{\operatorname{ArcTanh}\left[\frac{\omega_{D}}{\sqrt{\omega^{2}-\Delta^{2}}}\right]}{\sqrt{\omega^{2}-\Delta^{2}}}, & \omega^{2}>\Delta^{2}
\end{array}
$$

Infinite Debye frequency case:

The Green's function for the non-magnetic impurities, analytically is obtained by 


$$
G(r)=\lim _{t \rightarrow 0^{-}} G_{N M_{1}}(\vec{r}, t)=\left(\lim _{t \rightarrow 0^{-}} \int G^{(0)}(r, \omega) e^{i \omega t} d \omega\right) e^{\frac{-r}{2 \tau V_{F}}} \equiv\left(G^{(0)}(\vec{r})\right) e^{\frac{-r}{2 \tau V_{F}}}
$$

where $v_{F}$ is the Fermi velocity and $G^{(0)}(\vec{r})$ was given in Refs. [8-9]. For s-wave superconductors, the angular dependence doesn't exist in Green's Functions. It worth mentioning that Eq.(37) is equal the product of the Green's function of the pure superconductor and $\operatorname{Exp}\left(-r / 2 \tau v_{F}\right)$. Also, by considering the rare-earth metal impurity, up to the first-order approximation, the Green's functions are obtained as follows

$$
\begin{gathered}
G_{M_{1}}^{(1)}(\vec{k}, \omega)=\frac{\omega+\epsilon_{\vec{k}}+i / 2 \tau_{1}}{\left(\omega^{2}-\Delta^{2}-\epsilon_{\vec{k}}^{2}\right)}\left(1-\frac{\frac{i \omega}{\tau_{1}}-\frac{i|b| \Delta}{\tau_{2}}}{\left(\omega^{2}-\Delta^{2}-\epsilon_{\vec{k}}^{2}\right)}\right) ; \omega^{2}>\Delta^{2} \\
G_{M_{2}}^{(1)}(\vec{k}, \omega)=\frac{\omega+\epsilon_{\vec{k}}+|a| / 2 \tau_{1}}{\left(\omega^{2}-\Delta^{2}-\epsilon_{\vec{k}}^{2}\right)}\left(1-\frac{\frac{\omega|a|}{\tau_{1}}-\frac{\Delta}{\tau_{2}}}{\left(\omega^{2}-\Delta^{2}-\epsilon_{\vec{k}}^{2}\right)}\right) ; \Delta^{2}>\omega^{2}
\end{gathered}
$$

where $a^{2} \equiv \omega^{2} / \Delta^{2}$ and $b^{2} \equiv 1 / a^{2}$. After using the numerical calculations, $G_{M_{1,2}}(\vec{r})$ obtained.

Finite Debye frequency case:

For having a full discussion about finite Debye frequency, we try to compare the different circumstances of the $\omega, \Delta$, and $\omega_{D}$. In fact, the different condition leads to produce different $\eta_{\omega}$ s and subsequently, appropriate Green's functions for each condition are obtained. First, up to the first-order approximation, we investigate the Green's functions in the presence of the non-magnetic impurity with respect to the inverse collision time for different conditions.

The perturbed Green's function in the momentum-frequency space, under assumptions $\omega^{2}>\Delta^{2}$ and $\omega_{D}<\sqrt{\omega^{2}-\Delta^{2}}$, is obtained by

$$
G_{N M_{2}}^{(1)}(\vec{k}, \omega)=\frac{\omega}{\tau}\left(\frac{\omega_{D}\left(\omega^{2}-\Delta^{2}+\epsilon_{\vec{k}}^{2}\right)}{\pi\left(\omega^{2}-\Delta^{2}\right)\left(\omega^{2}-\Delta^{2}-\epsilon_{\vec{k}}^{2}\right)^{2}}\right)
$$

The perturbed Green's function at position space obtains by doing the integration of $G_{N M_{2}}^{(1)}(\vec{k}, \omega)$ with respect to the $\omega$ on two interval, $\omega<-\sqrt{\omega_{D}^{2}+\Delta^{2}}$ and $\omega>$ $\sqrt{\omega_{D}^{2}+\Delta^{2}}$.Then, after doing the Fourier transform between momentum space and position space, we obtain $G_{N M_{2}}^{(1)}(r)$. Subsequently, under assumptions $\omega^{2}>\Delta^{2}$ and $\omega_{D} \gg \sqrt{\omega^{2}-\Delta^{2}}$, we obtain 
and under assumptions $\omega^{2}<\Delta^{2}$ and $\omega_{D} \gg \sqrt{\Delta^{2}-\omega^{2}}$, we obtain

$$
G_{N M_{4}}^{(1)}(\vec{k}, \omega)=-\frac{1}{\tau}\left(\frac{\omega\left(-\pi \omega_{D}+2 \sqrt{-\omega^{2}+\Delta^{2}}\right)\left(\omega^{2}-\Delta^{2}+\epsilon_{\vec{k}}^{2}\right)}{2 \pi \omega_{D} \sqrt{-\omega^{2}+\Delta^{2}}\left(\omega^{2}-\Delta^{2}-\epsilon_{\vec{k}}^{2}\right)^{2}}\right)
$$

$G_{N M_{3}}^{(1)}(r)$ and $G_{N M_{4}}^{(1)}(r)$ are obtained, numerically, from Eqs.(41) and (42). The last condition that can be considered is $\omega^{2}<\Delta^{2}$ accompanied with $\omega_{D}<\sqrt{\Delta^{2}-\omega^{2}}$, does not show the physical result, because, it is in contrast with the statement that expresses always order parameter is less than the Debye frequency.

We now turn to the rare-earth metal impurity case. We investigate Green's functions in the presence of the rare-earth metal impurities, up to the first-order approximation with respect to $1 / \tau_{1}$ and $1 / \tau_{2}$ for different conditions. The perturbed Green's function under assumptions $\omega^{2}>\Delta^{2}$ and $\omega_{D}<\sqrt{\omega^{2}-\Delta^{2}}$, is obtained as follows

$$
G_{M_{3}}^{(1)}(\vec{k}, \omega)=\frac{\omega+\epsilon_{\vec{k}}-\omega_{D} / \tau_{1} \pi \omega}{\left(\omega^{2}-\Delta^{2}-\epsilon_{\vec{k}}^{2}\right)}\left(1-\frac{-\frac{2 \omega_{D}}{\tau_{1} \pi}+\frac{2 \omega_{D} b^{2}}{\tau_{2} \pi}}{\omega^{2}-\Delta^{2}-\epsilon_{\vec{k}}^{2}}\right)
$$

The perturbed Green's function at position space obtains by doing the integration of $G_{M_{3}}^{(1)}(\vec{k}, \omega)$ with respect to the $\omega$ on two interval, $\omega<-\sqrt{\omega_{D}^{2}+\Delta^{2}}$ and $\omega>$ $\sqrt{\omega_{D}^{2}+\Delta^{2}}$ and then, doing the Fourier transform between momentum space and position space. Subsequently, under condition with $\omega^{2}>\Delta^{2}$ and $\omega_{D} \gg \sqrt{\omega^{2}-\Delta^{2}}$, we obtain

$G_{M_{4}}^{(1)}(\vec{k}, \omega) \approx\left(\frac{1}{\omega^{2}-\Delta^{2}-\epsilon_{\vec{k}}^{2}}\right)\left(\left(\omega+\epsilon_{\vec{k}}\right)\left(1-\frac{\frac{1}{\tau_{1}}\left(i \omega-\frac{2 \omega^{2}}{\pi \omega_{D}}\right)+\frac{1}{\tau_{2}}\left(\frac{2 \Delta^{2}}{\pi \omega_{D}}-i|b| \Delta\right)}{\left(\omega^{2}-\Delta^{2}-\epsilon_{\vec{k}}^{2}\right)}\right)-\frac{\omega}{\pi \tau_{1} \omega_{D}}+\frac{i}{2 \tau_{1}}\right)$

And the perturbed Green's function with assumptions $\Delta^{2}>\omega^{2}$ and $\omega_{D} \gg \sqrt{\Delta^{2}-\omega^{2}}$ is $G_{M_{5}}^{(1)}(\vec{k}, \omega) \approx \frac{1}{\left(\omega^{2}-\Delta^{2}-\epsilon_{\vec{k}}^{2}\right)}\left(\left(\omega+\epsilon_{\vec{k}}\right)\left(1-\frac{\frac{1}{\tau_{1}}\left(|a| \omega-\frac{2 \omega^{2}}{\pi \omega_{D}}\right)+\frac{1}{\tau_{2}}\left(\frac{2 \Delta^{2}}{\pi \omega_{D}}-\Delta\right)}{\left(\omega^{2}-\Delta^{2}-\epsilon_{\vec{k}}^{2}\right)}\right)-\frac{\omega}{\pi \tau_{1} \omega_{D}}+\frac{|a|}{2 \tau_{1}}\right)$

And eventually, $G_{M_{4}}^{(1)}(r)$ and $G_{M_{5}}^{(1)}(r)$ are obtained from Eqs. (44) and (45). Although, basically, the above results are also obtained by the perturbed Abrikosov-Gorkov 
equations. By replacing the $G(\vec{k}, \omega)$ and $F^{\dagger}(\vec{k}, \omega)$ by $\left(G^{(0)}(\vec{k}, \omega)+G^{(1)}(\vec{k}, \omega)\right)$ and $\left(F^{(0)^{\dagger}}(\vec{k}, \omega)+F^{(1)^{\dagger}}(\vec{k}, \omega)\right)$, respectively, to the primary AG equations, the perturbed AG linearized equations in the presence of the impurity are obtained as follows

$$
\begin{gathered}
\left(\omega-\epsilon_{\vec{k}}\right) G^{(1)}(\vec{k}, \omega)-\bar{G}(\omega) G^{(0)}(\vec{k}, \omega)+\Delta{F^{(1)}}^{\dagger}(\vec{k}, \omega)+\bar{F}^{\dagger}(\omega) F^{(0)^{\dagger}}(\vec{k}, \omega)=0 \\
\left(\omega+\epsilon_{\vec{k}}\right) F^{(1)^{\dagger}}(\vec{k}, \omega)-\bar{G}(\omega) F^{(0)^{\dagger}}(\vec{k}, \omega)+\Delta G^{(1)}(\vec{k}, \omega)+\bar{F}^{\dagger}(\omega) G^{(0)}(\vec{k}, \omega)=0
\end{gathered}
$$

Then, $G^{(1)}(\vec{k}, \omega)$ is

$$
G^{(1)}(\vec{k}, \omega)=\left(\bar{G}(\omega)\left(\left(\omega+\epsilon_{\vec{k}}\right)^{2}+\Delta^{2}\right)+2 \Delta \bar{F}^{\dagger}(\omega)\left(\omega+\epsilon_{\vec{k}}\right)\right) /\left(\omega^{2}-\epsilon_{\vec{k}}^{2}-\Delta^{2}\right)^{2}
$$

As an instance for infinity value of the Debye frequency, $\bar{G}(\omega)$ and $\bar{F}^{\dagger}(\omega)$ are given by

$$
\bar{G}(\omega)=i \omega / 2 \tau_{1}\left(\omega^{2}-\Delta^{2}\right)^{1 / 2}, \bar{F}^{\dagger}(\omega)=-i \Delta / 2 \tau_{2}\left(\omega^{2}-\Delta^{2}\right)^{1 / 2}
$$

And again the different conditions of the $\omega$ and $\Delta$ lead to the different Green's functions.

\section{References}

1. Preskill, J. Quantum information and physics: some future directions. J. Mod. Opt. 47, 127-137 (2000)

2. Fetter, A. L. \& Walecka, J. D. Quantum Theory of Many Particle Systems (McGraw-Hill, New York, 1971).

3. Horodecki, R., Horodecki, P., Horodecki, M. \& Horodecki, K. Quantum entanglement. Rev. Mod. Phys. 81, 865-942 (2009).

4. Osterloh, A., Amico, L., Falci, G. \& Fazio, R. Scaling of entanglement close to a quantum phase transition. Nature 416, 608-610 (2002)

5. Osborne, T. J. \& Nielsen, M. A. Entanglement in a simple quantum phase transition. Phys. Rev. A 66, $032110(2002)$.

6. Vedral, V. Quantum entanglement. Nature Phys 10, 256-258 (2014).

7. Amico, L., Fazio, R., Osterloh, A. \&Vedral, V. Entanglement in many-body systems. Rev. Mod. Phys. 80, 517-576 (2008).

8. Oh, S. \& Kim, J. Entanglement of electron spins in superconductors. Phys. Rev. B 71, 144523-144526 (2005).

9. Afzali, R., Ebrahimian, N. \& Eghbalifar, B. Quantum information aspects on bulk and nano interacting Fermi system: A spin-space density matrix approach. Phys. Lett. A 380, 3394-3403 (2016).

10. Afzali, R., Fahimi, S. \& Dehghan, M. Quantum Entanglement and Correlation Lengths of a S-wave 
Superconductors in the Presence of a Weak Constant External Potential. International Journal of Theoretical Physics 56, 1565-1576 (2017).

11. Hill, S. \& Wootters, W. K. Entanglement of a pair of quantum bits. Phys. Rev. Lett. 78, 5022-5025 (1997).

12. Wootters, W. K. Entanglement of formation of an arbitrary state of two qubits. Phys. Rev. Lett. 80, 22452248 (1998).

13. Oh, S. \& Kim, J. Entanglement of electron spins of noninteracting electron gases. Phys. Rev. A69,054305 (2004).

14. Vedral, V. Entanglement in the second quantization formalism. Cent. Eur. J. Phys. 2, 289-306 (2003).

15. Lunkes, C., Brukner, Č. \& Vedral, V. Natural Multiparticle Entanglement in a Fermi Gas. Phys. Rev. Lett.95,030503 (2005).

16. Wu, L.-A., Sarandy, M. S. \& Lidar, D. A. Quantum phase transitions and bipartite entanglement. Phys. Rev. Lett. 93, 250404 (2004).

17. Chiara, G. D. \& Sanpera, A. Genuine quantum correlations in quantum many-body systems: a review of recent progress. Rep. Prog. Phys.81, 074002 (2018).

18. Yang, M.-F. Reexamination of entanglement and the quantum phase transition. Phys. Rev. A71, (2005).

19. Balatsky, A. V., Vekhter, I. \& Zhu, J-X. Impurity-induced states in conventional and unconventional superconductors. Rev. Mod. Phys. 78, 373-433 (2006).

20. Crişan, M. Theory of superconductivity. (World Scientific Publishing, Singapore 1989).

21. Bennemann, K. H., Ketterson, J. B., eds. Superconductivity: Conventional and Unconventional Superconductors Vol. 1 (Springer, Berlin, 2008).

22. De Gennes, P. G. Superconductivity of metals and alloys (W. A. Benjamin, New York, 1966).

23. Anderson, P. W. Theory of dirty superconductors. J. Phys. Chem. Solids 11, 26-30 (1959).

24. Abrikosov, A. A. \&Gorkov, L. P. On the theory of superconducting alloys.1. The electrodynamics of alloys at absolute zero. Sov. Phys. JETP 8, 1090-1098 (1959).

25. Soda, T., Matsuura, T. \& Nagaoka, Y. s-d Exchange Interaction in a Superconductor. Prog. Theor. Phys. 38, 551-567 (1967).

26. Shiba, H. Classical spins in superconductors. Prog. Theor. Phys. 40, 435-451 (1968).

27. Rusinov, A. I. Theory of gapless superconductivity in alloys containing paramagnetic impurities. Sov. Phys. JETP 29, 1101-1106 (1969).

28. Okabe, Y. \& Nagi, A. D. S. Role of potential scattering in the Shiba-Rusinov theory of the magnetic impurities in superconductors. Phys. Rev. B 28, 1320-1322 (1983).

29. Okabe, Y. \& Nagi, A. D. S. Anisotropic superconductors containing paramagnetic impurities. Phys. Rev. $B$ 28, 1323-1328 (1983).

30. Chaba, A. N. \& Nagi, A. D. S. Properties of Superconducting Alloys Containing Paramagnetic Impurities with Local States within the Gap. Can. J. Phys. 50, 1736-1746 (1972).

31. Warier, K. B. \& Nagi, A. D. S. Effect of gap anisotropy in superconductors containing paramagnetic impurities with local states within the gap. J. Low Temp. Phys. 45, 97-108 (1981).

32. Lo, S. C. \& Nagi, A. D. S. Josephson tunnel effect and the order parameter of superconducting alloys 
containing paramagnetic impurities with local states within the gap. Phys. Rev. B9, 2090-2096 (1974).

33. Sihota, B. \& Nagi, A. D. S. Effect of a magnetic field on the specific heat jump of a superconducting alloy containing paramagnetic impurities with local states within the gap. J. Low Temp. Phys. 51, 347368 (1983).

34. Sau, J. D. \& Demler, E. Bound states at impurities as a probe of topological superconductivity in nanowires. Phys. Rev. B88,205402 (2013).

35. Körber, S., Trauzettel, B. \& Kashuba, O. Collective Yu-Shiba-Rusinov states in magnetic clusters at superconducting surfaces. Phys. Rev. B97, 184503 (2018).

36. Wu, C.-T., Setiawan, F., Anderson, B. M., Hsiao, W.-H. \& Levin, K. Quantum phase transitions in proximitized Josephson junctions. Phys. Rev B98,064504 (2018).

37. Rouco, M., Tokatly, I. V. \& Bergeret, F. S. Spectral properties and quantum phase transitions in superconducting junctions with a ferromagnetic link. Phys. Rev. B99, 094514(2019).

38. Farinacci, L. et al. Tuning the Coupling of an Individual Magnetic Impurity to a Superconductor: Quantum Phase Transition and Transport. Phys. Rev. Lett. 121,196803(2018).

39. Sacramento, P. D., Nogueira, P., Vieira, V. R. \& Dugaev, V. K. Entanglement signatures of the quantum phase transition induced by a magnetic impurity in a superconductor. Phys. Rev. B 76, 184517(2007).

40. Głodzik, S. \& Ptok, A. Quantum Phase Transition Induced by Magnetic Impurity. J. Supercond. Nov. Magn. 31, 647-650 (2017).

41. Fulde, P. Effects of Gap Anisotropy in Superconductors Containing Paramagnetic Impurities. Phys. Rev. 139, A726(1965).

42. Feenberg, E. Ground state of an interacting boson system. Ann. Phys. (N. Y.) 84, 128-146 (1974).

43. Clark, J. W., Habibian, H., Mandilara, A. D. \& Ristig, M. L. Aspects of Entanglement in Quantum Many-Body Systems. Found. Phys.40, 1200-1220 (2010).

44. Skalski, S., O. Betbeder-Matibet, and P. R. Weiss. Properties of superconducting alloys containing paramagnetic impurities. Phys. Rev. 136,6A, A1500 (1964).

45. Openov, Leonid A. Effect of nonmagnetic and magnetic impurities on the specific heat jump in anisotropic superconductors. Phys. Rev. B 69, 224516 (2004).

46. Kümmel, Reiner. Electronic structure of superconductors with dilute magnetic impurities. Phys. Rev. B 6, 2617 (1972).

47. Tsuzuki, Toshio, and Toshihiko Tsuneto. Spatial Variation of the Order Parameter in the Vicinity of a Paramagnetic Impurity. Prog. Theor. Phys.37, 1-12 (1967).

48. Zhitomirsky, M. E., and M. B. Walker. "Effect of spatial variations of the superconducting gap on suppression of the transition temperature by impurities." Phys. Rev. Lett. 80,5413 (1998).

49. Afzali, R., A. T. Rezakhani, and H. R. Alborznia. Effect of weak magnetic field on quantum spin correlation in an s-wave superconductor. arXiv preprint arXiv:1011.3011 (2010).

50. Kunz, A. B., and D. M. Ginsberg. Band calculation of the effect of magnetic impurity atoms on the properties of superconductors. Phys. Rev. B 22,3165 (1980).

51. Boato, G., G. Gallinaro, and C. Rizzuto. Effect of transition-metal impurities on the critical temperature of superconducting Al, Zn, In, and Sn. Phys. Rev. 148,353 (1966). 
52. Collings, E. W., F. T. Hedgcock, and Y. Muto. Role of $\mathrm{s}^{-} \mathrm{d}$ Exchange Interactions in Dilute Alloys Exhibiting Both Low-Temperature Resistance Anomalies and Superconductivity. Phys. Rev. 134,6A, A1521 (1964).

53. Leon, B., and AD Singh Nagi. Calculation of the electronic thermal conductivity of superconducting alloys with paramagnetic impurities. J. Phys. F: Met. Phys. 5,1533 (1975).

54. Lemberger, Thomas R., and D. M. Ginsberg. Comparison of the thermal conductivity of superconducting Zn-Mn alloys with Shiba's theory. Phys. Rev. B 14,1785 (1976).

55. Suhl, Harry, and George Tibor Rado, eds. Magnetism: a treatise on modern theory and materials. 5. Magnetic properties of metallic alloys. (Academic Press, Massachusetts,1973).

\section{Acknowledgements}

The authors are grateful for the helpful discussion with Dr. habil. Andreas Osterloh

\section{Author Contributions}

All authors equally performed the calculations, discussed the results, and participated in writing and reviewing the manuscript. R. A. gives the idea of this research. 
Figures

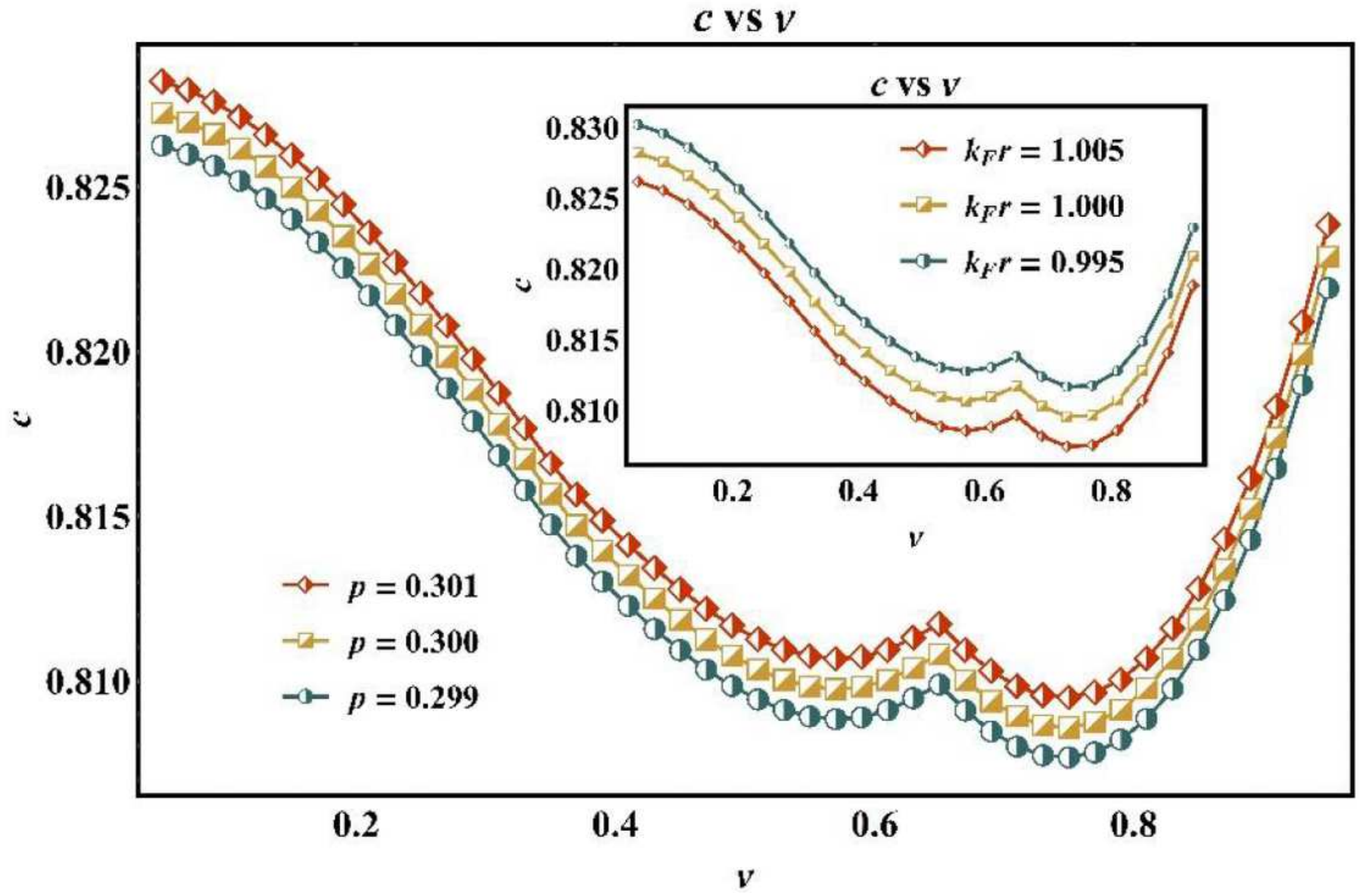

Figure 1

For transition-metal case; concurrence versus $v$ for various values of the $p$ at $j=-0.8$ and $k F r=1$. Inset: concurrence versus $v$ for various values of the $k F r$ at $j=-0.8$ and $p=0.3$. 


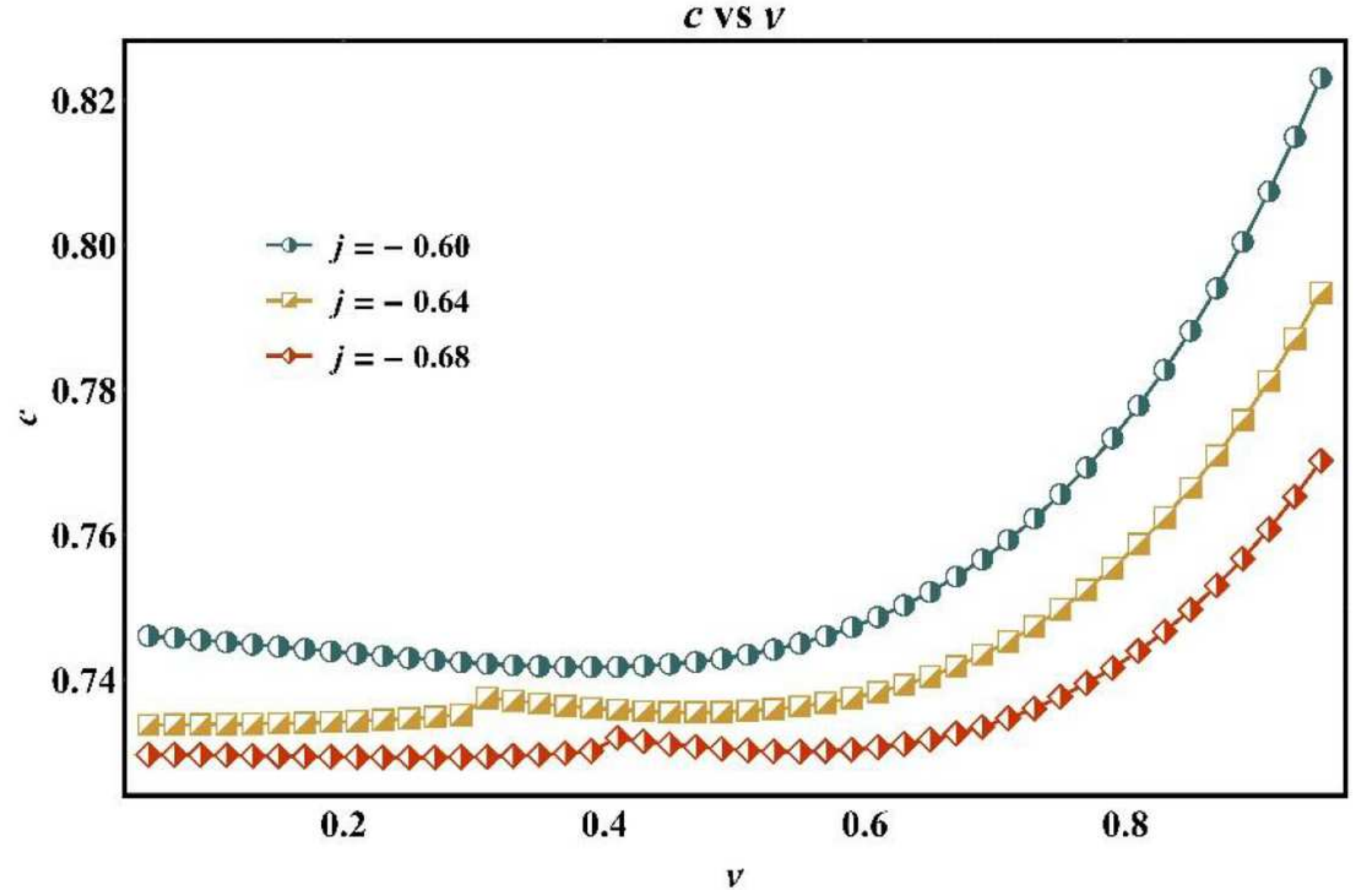

Figure 2

For transition-metal case; concurrence versus $v$ for various values of the $\mathrm{j}$ at $\mathrm{p}=0.2$ and $\mathrm{kFr}=1$. 


\section{$c$ VS $v$}

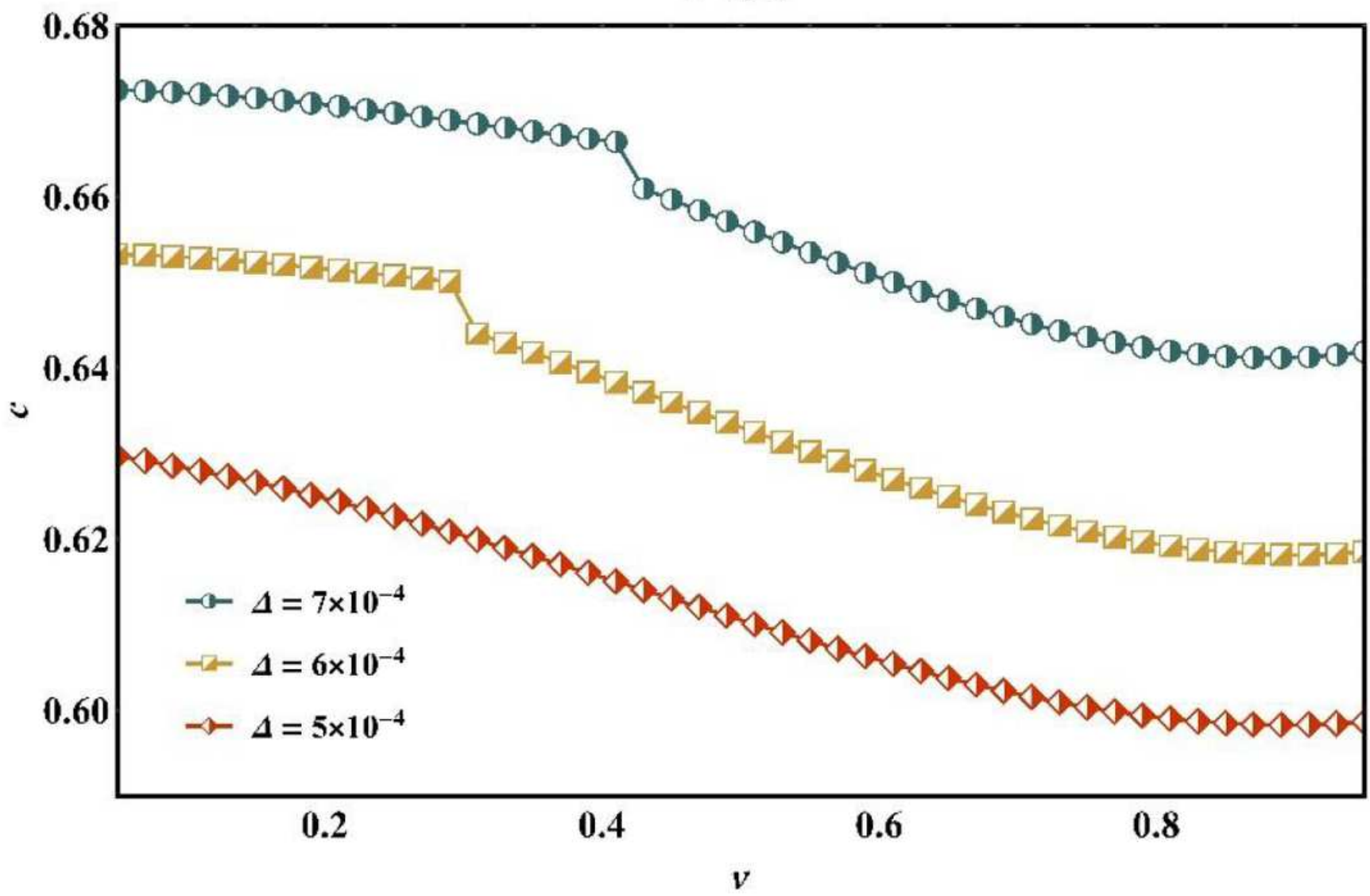

Figure 3

For transition metal case; concurrence versus $v$ for various values of the $\Delta$ at $j=-0.8, p=0.2$ and $k F r=1$.
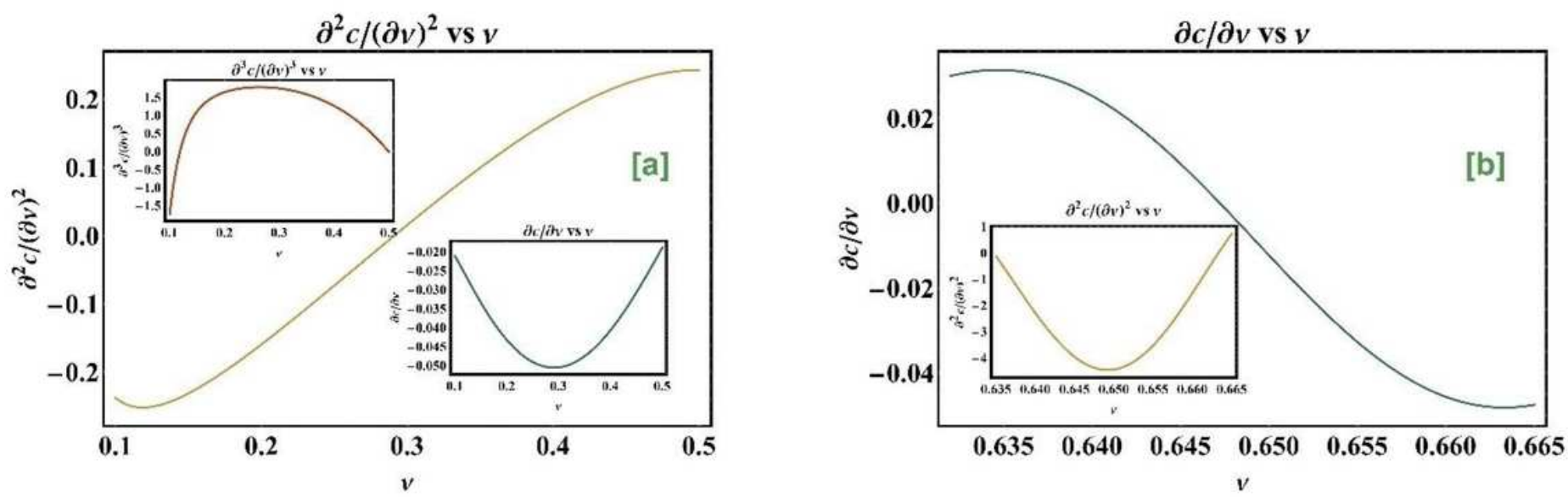

Figure 4

partial derivatives of $c$ vs vat $j=-0.8, k F r=1$ and $p=0.3(a)$ : for each $v$ that located in the interval $(0.1,0.5)$ (b): for each $v$ that located in the interval $(0.635,0.665)$. 


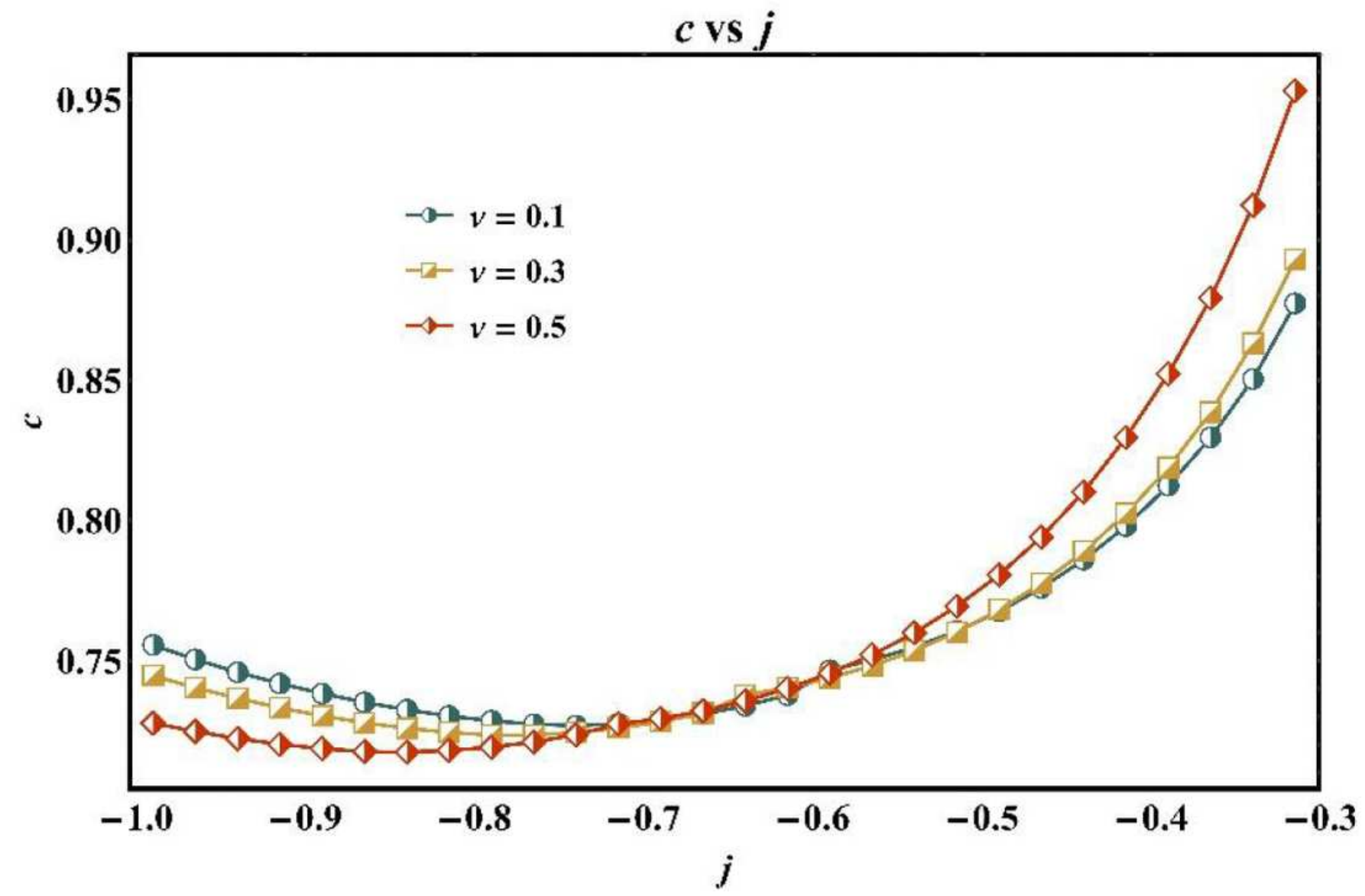

Figure 5

for transition-metal case; concurrence versus $\mathrm{j}$ for various values of the vat $\mathrm{p}=0.2$ and $\mathrm{kFr}=1$. 


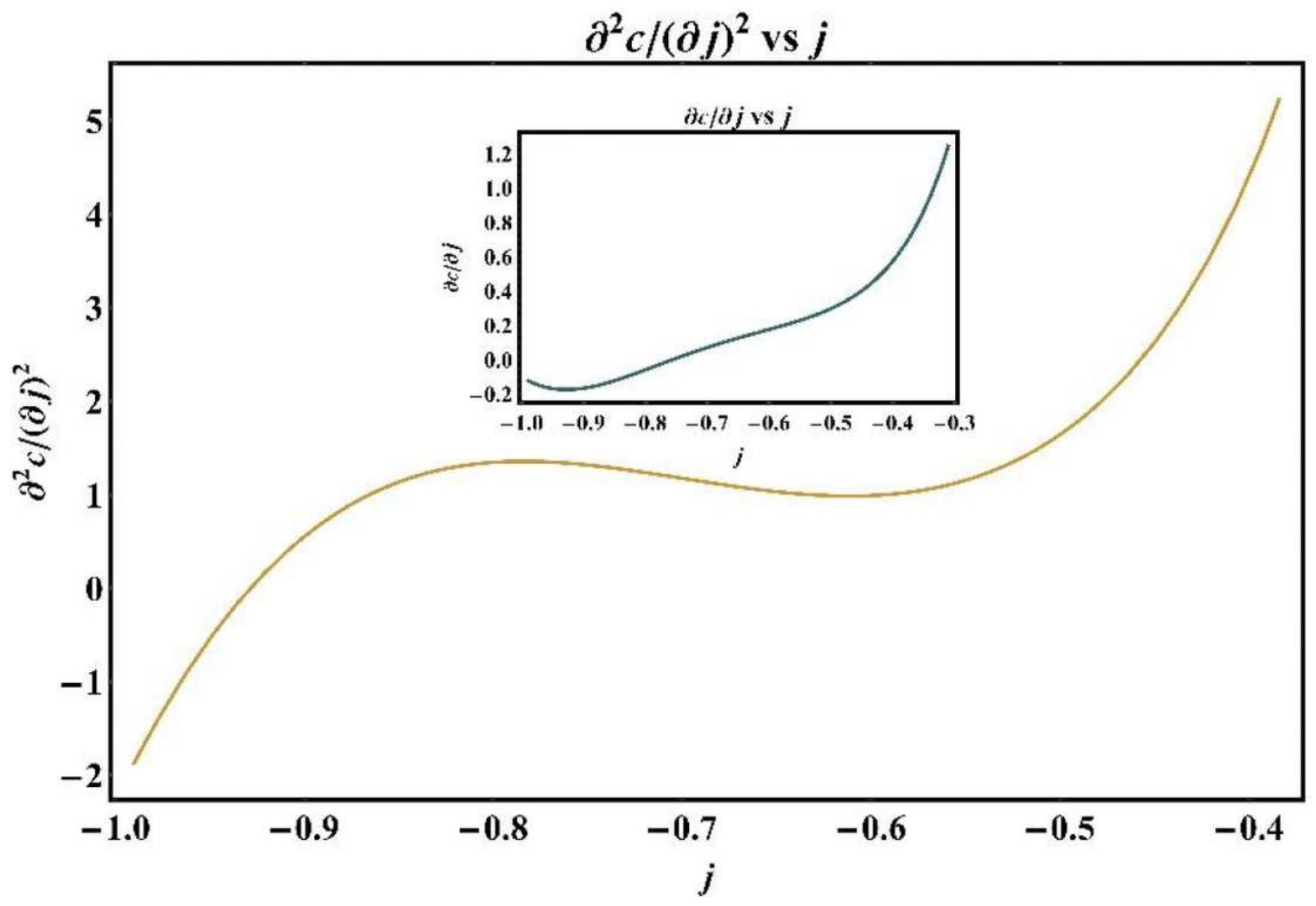

Figure 6

partial derivatives of $\mathrm{c}$ versus $\mathrm{f}$ at $\mathrm{v}=0.1, \mathrm{kFr}=1$ and $\mathrm{p}=0.2$.
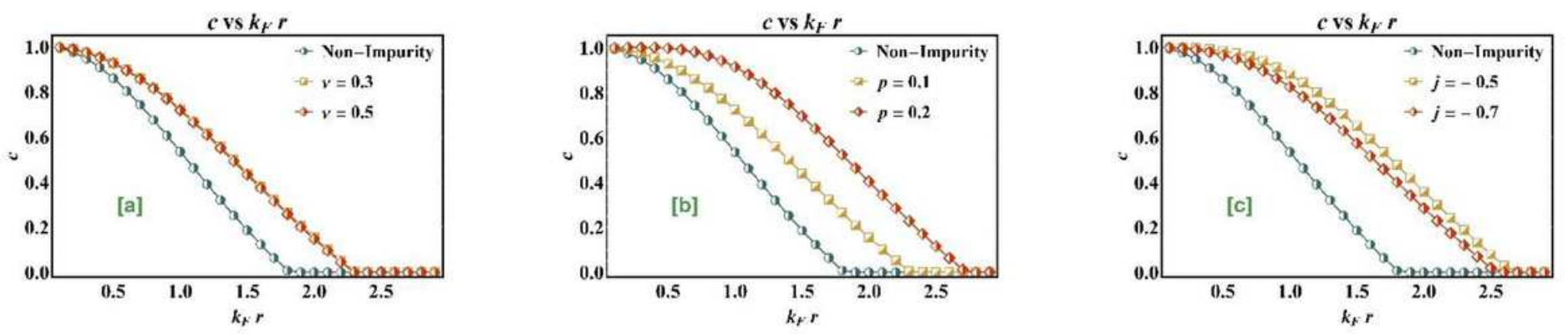

Figure 7

(a): for transition-metal case; concurrence versus $\mathrm{kFr}$ for various values of $v$ at $\mathrm{j}=-0.8$ and $p=0.2$. (b): concurrence versus $\mathrm{kFr}$ for various values of pat $\mathrm{j}=-0.3$ and $\mathrm{v}=0.3$. (c): concurrence versus $\mathrm{kFr}$ for various values of $j$ at $p=0.3$ and $v=0.3$. 

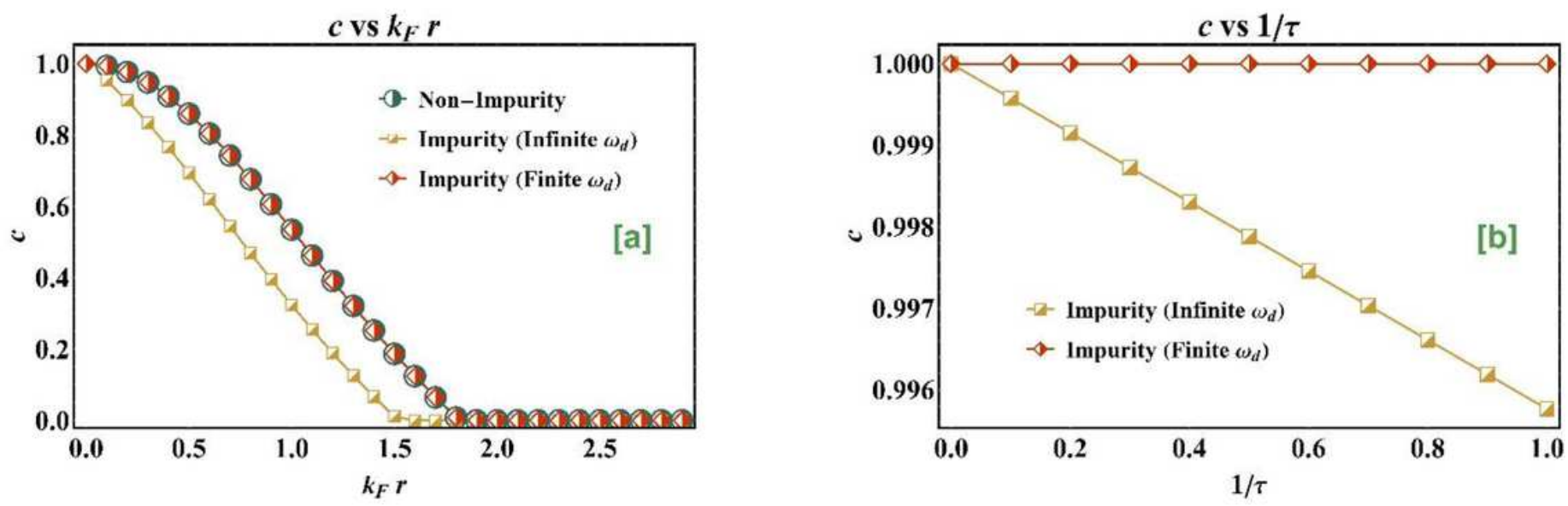

Figure 8

(a): for Rare-earth metal case; concurrence versus kFr for infinite and finite Debye frequency in the presence of the impurity and non-impurity cases. (b): concurrence versus inverse collision time for infinite and finite Debye frequency in the presence of the impurity and non-impurity cases.

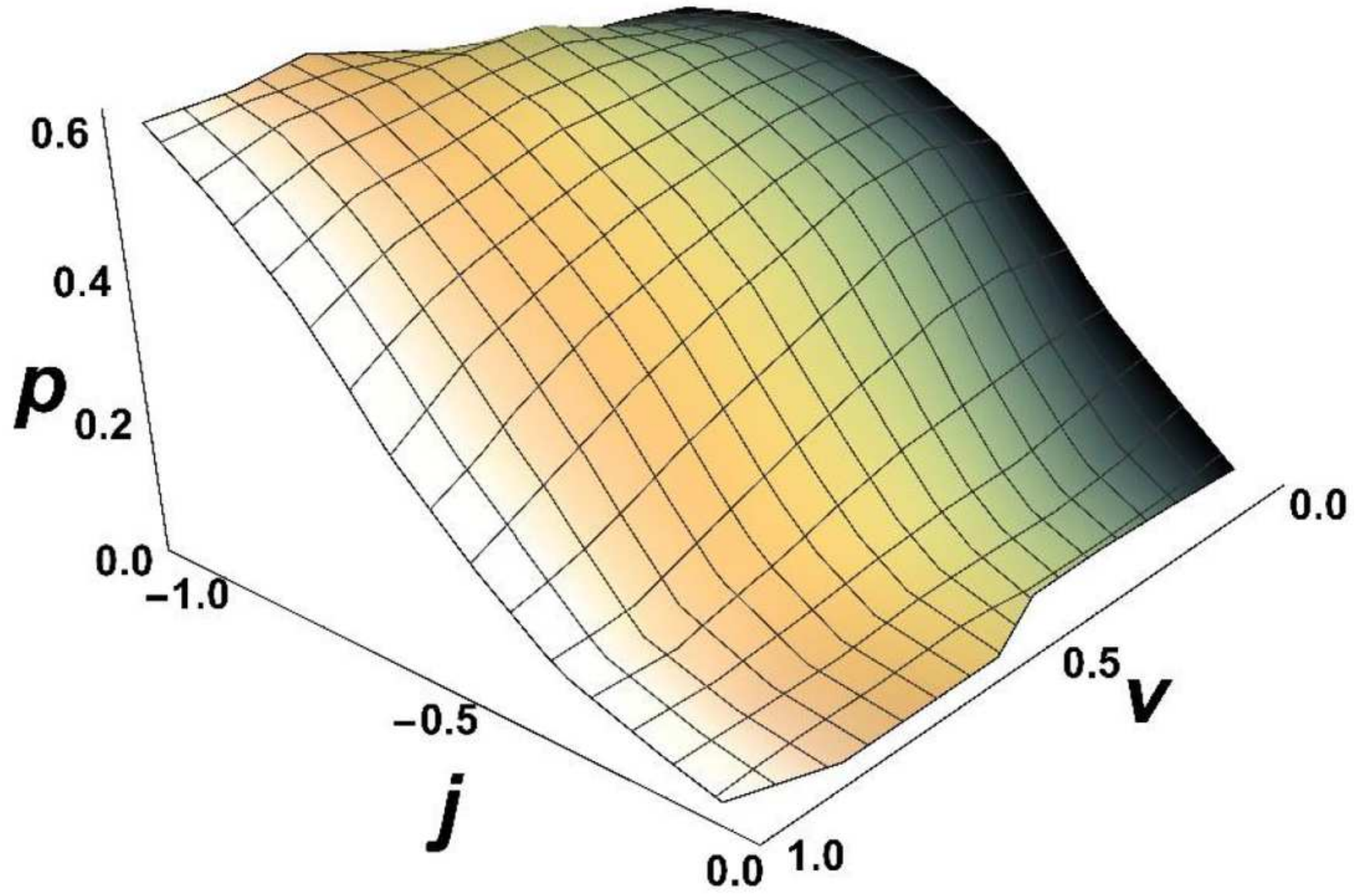

Figure 9 
The permissible range (under the manifold) of $j, v$ and $p$ with respect to the low value of the $\operatorname{Im}[G(1)$ $(\mathrm{r})] / \operatorname{Im}[\mathrm{G}(0)(0)]$ 